\title{
Collective organization and screening in two-dimensional turbulence
}

\author{
Javier Jiménez ${ }^{*} *$ \\ School of Aeronautics, Universidad Politécnica de Madrid, 28040 Madrid, Spain
}

(Received 14 March 2021; accepted 1 July 2021; published 2 August 2021)

\begin{abstract}
Following recent evidence that the vortices in decaying two-dimensional turbulence can be classified into small mobile and large quasistationary, this paper examines the evidence that the latter might be considered a crystal whose formation embodies the inverse cascade of energy towards larger scales. Several diagnostics of order are applied to the ostensibly disordered large vortices. It is shown that their geometric arrangement is substantially more regular than random, that they move more slowly than could be expected from simple mean-field arguments, and that their energy is significantly lower than in a random reorganization of the same vortices. This is traced to screening of long-range interactions by the preferential association of vortices of opposite sign and it is argued that this is due to the mutual capture of corotating vortices, in a mechanism closer to tidal disruption than to electrostatic screening. Finally, the possible relation of these stochastic crystals to fixed points of the dynamical system representation of the turbulence flow is briefly examined.
\end{abstract}

DOI: 10.1103/PhysRevFluids.6.084601

\section{INTRODUCTION}

This paper is part of a sequence that began as an investigation on whether an automatic computer search of relevant features in decaying two-dimensional turbulence could be used to suggest new ideas about the organization of the flow [1]. That paper was continued in [2,3], mostly from the point of view of how such a search should be organized and of its possible relation to automatic learning, and eventually led in [4] to an analysis of the flow itself. It is with the conclusions of this last paper that the present one deals.

Two-dimensional turbulence is a well-studied phenomenon, often used as an approximation to geophysical and stratified flows in which isotropy is broken by some constraint along the third dimension. Although truly two-dimensional turbulence is experimentally challenging, it was one of the first turbulent flows to become accessible to direct numerical simulation, and computational experiments were soon undertaken to test theoretical predictions [5-9]. Much of its theoretical interest can be traced to the early observation in [10] that a system of point vortices in a plane could lead, under some conditions, to states of negative temperature and to the formation of large-scale structures. We will see later that the point-vortex model is a poor approximation to turbulence, because the former is Hamiltonian and conserves enstrophy (the square of vorticity) and kinetic energy, while the latter is dissipative, but the statistical mechanics of point vortices has continued to be examined in the hope that it may be locally relevant to the dissipative case $[6,11,12]$. Dissipative mean-field theories based on cascades of the inviscid conserved quantities were developed almost

\footnotetext{
*jjsendin@gmail.com
}

Published by the American Physical Society under the terms of the Creative Commons Attribution 4.0 International license. Further distribution of this work must maintain attribution to the author(s) and the published article's title, journal citation, and DOI. 
simultaneously to the Hamiltonian model [5,13], eventually leading to the prediction of a direct cascade of enstrophy towards smaller scales and an inverse cascade of energy towards larger ones $[13,14]$. The latter could intuitively be related to the negative-temperature states of [10].

However, simulations showed that the flow spontaneously segregates into coherent vortices and a less-coherent background and that the vortices interfere with the conclusions of the mean-field representation. By the end of the 1990s there was widespread consensus that two-dimensional turbulence is a vortex gas, following approximately Hamiltonian dynamics punctuated by occasional merging of vortices of like sign. The forward enstrophy cascade proceeds by successive vortex merging and filamentation [15-18] and is not described well by mean-field theories. The mechanism of the backward energy cascade is less clear, although it is not believed to be associated with the formation of larger vortices by amalgamation [19-22], but the phenomenon itself has been observed numerically, especially in forced systems kept in statistical equilibrium by a large-scale dissipation mechanism $[23,24]$. In a finite domain and in the absence of a dissipation mechanism, the energy accumulates at the largest available flow scale, in a phenomenon akin to Bose-Einstein condensation $[25,26]$ and to the predictions in [10]. Fuller reviews of previous work on two-dimensional turbulence can be found in $[27,28]$.

The results in [4] extended the previous models in some interesting directions, especially regarding the decay of unforced turbulence in the early period in which the spectral scale of the energy has not grown enough to be affected by the simulation domain. It was found that the largest structures of the kinetic energy take the form of elongated high-speed jets, flanked by a subset of large vortices that move relatively slowly with respect to the global root-mean-square (rms) velocity. The cascade of energy towards large scales corresponds to the growth of these jets. It was speculated in [4] that the slowly moving vortex dipoles that flank the jets form a large-scale collective structure that can approximately be described as a crystal and it was further speculated that the formation of the dipoles responds to a tendency of the flow to organize into low-energy configurations. This will be shown below to be the case, even if it may be considered counterintuitive in a system, like two-dimensional turbulence, which dissipates enstrophy rather than energy and in which the latter could be expected to lag behind the decay of the former.

The questions addressed in the present paper concern this possible collective structure. After describing in Sec. II the generation of the data and their general properties, we examine in Sec. III whether the flow really is in a low-energy state and whether this is connected with the presence of dipoles. Section IV looks at the question of vortex mobility. Sections V and VII discuss whether the organization of the large vortices is regular enough to be considered a crystal, even approximately.

Two related questions are addressed in the context of the previous results. The first one is whether there is any screening between vortices of different sign, as originally suggested by [29], and the second one is whether the vortex crystal described above could be related to the fixed-point solutions that have been invoked as organizing states for high-dimensional or turbulent dynamical systems [30]. A discussion of these and other questions is offered in Sec. VI. Section VIII provides a summary and discusses conclusions.

\section{NUMERICAL EXPERIMENTS}

The data used in this paper are mostly those in [4], to which the reader is referred for details. Simulations of decaying nominally isotropic two-dimensional turbulence are performed in a doubly periodic square box of side $L$, using a standard spectral Fourier code dealiased by the $2 / 3$ rule. Time advances via the third-order Runge-Kutta method. The flow field is defined by its velocity $\boldsymbol{u}=(u, v)$ in the $\boldsymbol{x}=(x, y)$ plane and by the one-component vorticity $\omega=\boldsymbol{\nabla} \times \boldsymbol{u}$. It is initialized with random Fourier phases and a fixed isotropic enstrophy spectrum whose peak, approximately located at wave number $k_{\text {init }}=2 \pi / L_{\text {init }}$, controls the initial width of the energy-containing spectral range. The equations are solved in a vorticity-stream-function formulation, using regular second-order viscosity $v \nabla^{2} \omega$, and the flow is evolved for a fixed time to allow vortical structures to establish themselves. This moment is defined as $t=0$ and denoted by a 0 subindex in the corresponding 
(a)

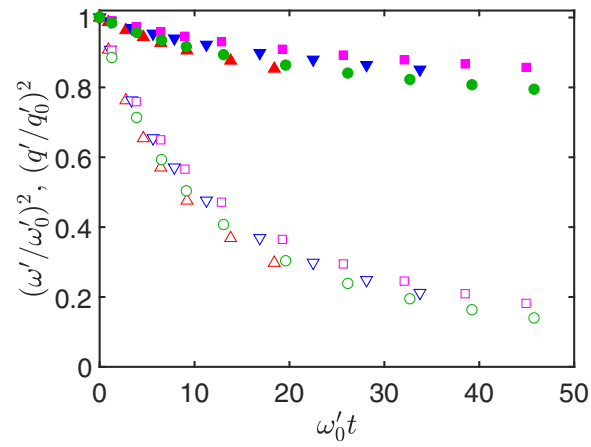

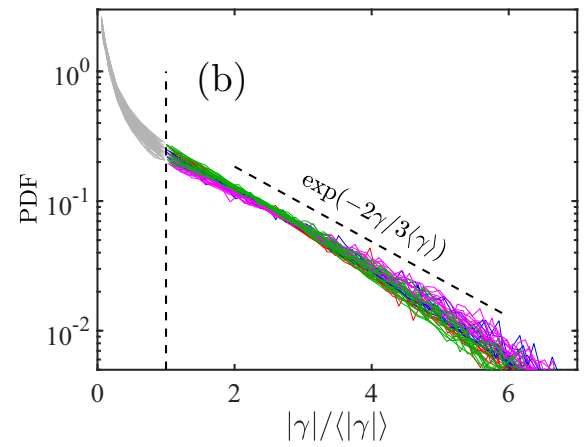

(c)

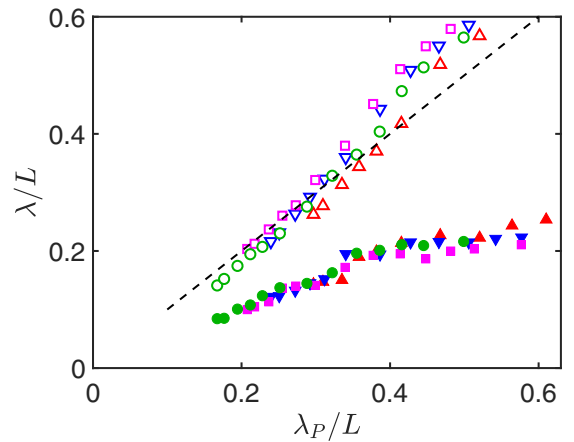

FIG. 1. (a) Evolution of the enstrophy (open symbols) and energy (closed symbols) of the simulations in Table I. Symbols are defined in the table. The colors denote the following: red, T512; blue, T768; magenta, T1024; and green, T1024a. They are used whenever possible throughout the paper. (b) Probability density functions of the vortex circulation. Only vortices with $\gamma /\langle\gamma\rangle>1$ are included in the reconstructed flow fields. (c) Comparison of the Poisson wavelength (3), with the spectral wavelengths $\lambda_{q}$ (open symbols) and $\lambda_{\omega}$ (closed symbols). The dashed line is $\lambda_{q}=\lambda_{P}$. (d) Open symbols are the mean distances between neighboring large vortices and closed symbols are the mean diameters of the large vortices, both versus $\lambda_{P} / L$. The dashed line is $\langle d\rangle=0.27 \lambda_{P}$ and the solid one is $\left\langle s^{1 / 2}\right\rangle=0.21 \lambda_{P}$.

quantities. Experiments are run as ensembles of at least 768 flows, with more samples added in some cases to improve statistics. Case T1024a, which was not in [4], has been included to provide a wider initial range of scales. As the simulation proceeds, the enstrophy $\left\langle\omega^{2}\right\rangle$, where the time-dependent average $\langle\cdot\rangle$ is taken over the full computational box and over the ensemble, decays by approximately 70-80\%, while the kinetic energy $\left\langle|\boldsymbol{u}|^{2}\right\rangle$ decreases by at most $15-20 \%$ [Fig. 1(a)]. The numerical parameters for the different cases are summarized in Table I. The time step is controlled by a Courant-Friedrichs-Lewy condition $N|\boldsymbol{u}|_{\max } \Delta t / 3=0.5$ and T1024a was repeated with a half as long time step to confirm the numerics.

Natural time and velocity scales can be defined from the rms vorticity magnitude $\omega^{\prime}=\left\langle\omega^{2}\right\rangle^{1 / 2}$ and from $q^{\prime}=\left(u^{\prime 2}+v^{\prime 2}\right)^{1 / 2}$. The two length scales mainly used in [4] are the energy spectral wavelength $\lambda_{q}=2 \pi / k_{q}$, defined by the maximum of the premultiplied energy spectrum $k E_{q q}(k)$, where $k$ is the wave-number magnitude, and the enstrophy wavelength $\lambda_{\omega}$, similarly defined from the enstrophy spectrum $k E_{\omega \omega}$. Both scales increase with time, but $\lambda_{q}$ increases faster than $\lambda_{\omega}$, reflecting the energy flow towards larger scales [13]. They are not proportional to each other and describe different flow features. It was shown in [4] that $\lambda_{\omega}$ is proportional to the diameter of individual vortices, defined as connected objects in which the vorticity magnitude is larger than $\omega^{\prime}$, while $\lambda_{q}$ measures the average distance $d$ between closest neighboring vortices of similar size. Our simulations are discontinued when $\lambda_{q} / L \approx 0.6$, beyond which the flow is considered to enter a different decay regime in which the energy transfer towards larger scales is substantially modified by 
TABLE I. Parameters of the simulations. The size of the doubly periodic computational box is $L \times L$. The rms vorticity $\omega_{0}^{\prime}$ and the velocity magnitude $q_{0}^{\prime}$ are measured after the initial discarded transient, decaying from an initial enstrophy spectrum whose peak is at wavelength $L_{\text {init }}$. The enstrophy and energy scales $\lambda_{\omega 0}$ and $\lambda_{q 0}$, respectively, are defined in the text. The number of collocation points used for dealiasing is $N \times N$. The subscript $F$ refers to the end of each simulation and the decay time after the initial transient $t_{F}$ is chosen so that $\lambda_{q F} / L \approx 0.6$. Each case is an ensemble of $N_{t}$ independent experiments. For details, see [4]. (The penultimate and antepenultimate columns in this table were wrong in [4]. The present ones are believed to be correct.)

\begin{tabular}{lcccccccccc}
\hline \hline Case & $N$ & $L_{\text {init }} / L$ & $q_{0}^{\prime} L / \nu$ & $N_{t}$ & $\lambda_{\omega 0} / L$ & $\lambda_{q 0} / L$ & $\omega_{0}^{\prime} t_{F}$ & $\omega_{F}^{\prime} / \omega_{0}^{\prime}$ & $q_{F}^{\prime} / q_{0}^{\prime}$ & Symbol \\
\hline T512 & 512 & 0.05 & 4400 & 768 & 0.129 & 0.256 & 23.0 & 0.50 & 0.91 & $\Delta$ \\
T768 & 768 & 0.025 & 7800 & 768 & 0.108 & 0.217 & 39.4 & 0.49 & 0.92 & $\nabla$ \\
T1024 & 1024 & 0.033 & 11000 & 1392 & 0.095 & 0.197 & 51.3 & 0.40 & 0.92 & $\square$ \\
T1024a & 1024 & 0.01 & 8900 & 2304 & 0.078 & 0.142 & 65.4 & 0.31 & 0.87 & $\circ$ \\
\hline \hline
\end{tabular}

the finite box. As already mentioned in the Introduction, vortices naturally separate into two classes. Those whose circulation $\gamma_{i}$ is weaker than the mean $\left|\gamma_{i}\right|<\left\langle\left|\gamma_{i}\right|\right\rangle$ tend to move fast, have a power-law distribution of area and circulation, and are only responsible for a small fraction of the total kinetic energy of the flow [4]. Vortices stronger than the mean move more slowly, have an exponential probability distribution [Fig. 1(b)], and are responsible for most of the kinetic energy. This was interpreted in [4] to mean that the large vortices form a crystal in approximate equilibrium, which is the subject of the present paper. From now on, although the simulations are always run without truncation, our analysis refers to the fraction of the flow reconstructed from the large vortices, once the smaller ones have been deleted.

Deleting vorticity from a periodic flow usually requires some correction, because periodicity of the velocity field is only possible if the overall circulation vanishes,

$$
\sum_{i} \gamma_{i}=0
$$

When this is not satisfied, it has to be compensated by a background uniform vorticity $-\sum \gamma_{i} / L^{2}$. We will see below that the total kinetic energy of the flow is proportional to the integrated squared circulation [31], so the expected relative error of the energy estimates in the following section is of the order of $\left(\sum \gamma_{i}\right)^{2} / \sum \gamma_{i}^{2}$. In our reconstructed flows, it is at most $O\left(10^{-4}\right)$.

It follows from the proportionality between $d$ and $\lambda_{q}$ that the average number of large vortices per flow field should decay as $\left(\lambda_{q} / L\right)^{-2}$. Empirically,

$$
n_{v} \approx 4\left(\frac{L}{\lambda_{q}}\right)^{2},
$$

which decays from approximately 150 to 15 in our simulations. The last number approximately agrees with [8], which studied similar flows at longer decay times, where $\lambda_{q}$ saturates to approximately $L$, and reported that the motion of the 17 largest vortices was approximately independent of their background. The number of small vortices is approximately twice higher than the number of those larger than the mean. Because counting large vortices is a more stable operation than finding the maximum of a noisy spectrum, especially in the late stages of the evolution in which the definition of $\lambda_{q}$ depends on a few low-wave-number harmonics, we will use in the following a Poisson wavelength inspired by (2),

$$
\frac{\lambda_{P}}{L}=2 n_{v}^{-1 / 2}
$$

The relation between $\lambda_{P}$ and the two spectral wavelengths is shown in Fig. 1(c). Figure 1(d) shows its relation to the mean distance between large vortices and to their diameter. It is not surprising 
(a)

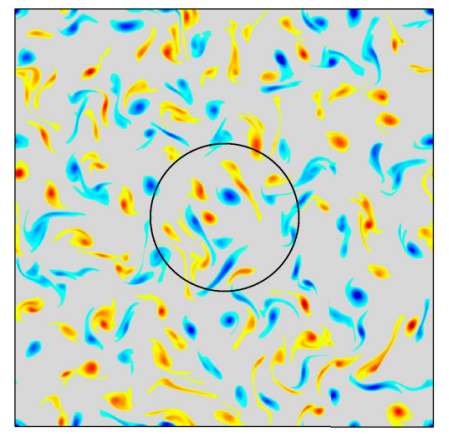

(c)

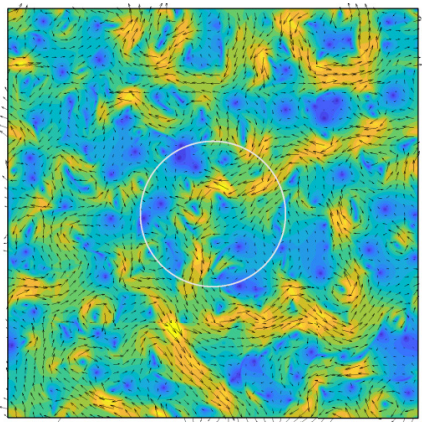

(b)

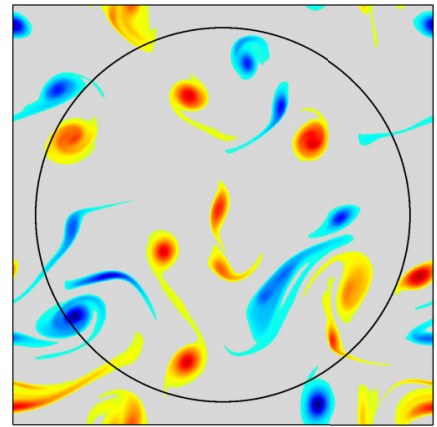

(d)

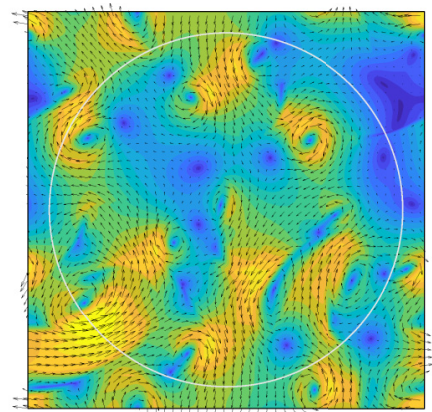

FIG. 2. (a) and (b) Flow vorticity synthesized by retaining only vortices whose area is larger than the average, in one of the members of the T1024a ensemble, for (a) $\omega_{0}^{\prime} t=1.3$ and $\lambda_{P} / L=0.18$ and (b) $\omega_{0}^{\prime} t=52.3$ and $\lambda_{P} / L=0.45$. The circle in each case has radius $\lambda_{P}$. (c) and (d) Velocity field for the flows in (a) and (b), respectively. The background color is the velocity magnitude, lighter for faster velocities.

that $\lambda_{P}$ is proportional to the distance between vortices, because this is how it is defined. In fact, assuming that the vortices are a Poisson-distributed set of points with density $n_{v} / L^{2}$, their average separation would be $\langle d\rangle=0.5 L / n_{v}^{1 / 2}=0.25 \lambda_{P}$ [4]. This linear dependence and even its coefficient are well satisfied in Fig. 1(d).

More interesting is that $\lambda_{P}$ is also linearly related to the vortex diameter $\left\langle s^{1 / 2}\right\rangle$, where $s$ is the area of the thresholded vortices. This was not the case for the full vortex system analyzed in [4], where the diameter was found to be proportional to $\lambda_{\omega}$, which is only approximately proportional to $\lambda_{q}$. Figure 1(d) implies that, even if the decay of the full flow is not self-similar in this way, the decay of the system of large vortices is truly self-similar, with a vortex diameter proportional to the separation. Two snapshots of the temporal evolution of the large-vortex component of a turbulent flow are shown in Fig. 2. The circles in the figure have radius $\lambda_{P}$ and (3) implies the number of vortices within them should stay approximately equal to $4 \pi$. The figure also displays the velocity field of these two flows, clearly showing the high-velocity streams mentioned in the Introduction [4].

Although not discussed in detail in this paper, reference will occasionally be made to simulations of Hamiltonian systems of point vortices. Their characteristics and numerical details are described in Appendix A.

\section{KINETIC ENERGY}

The clearest indication that the arrangement of the large vortices is not random is Fig. 3, which compares the total kinetic energy of an ensemble of large-vortex reconstructions of turbulent flows with that of another ensemble in which the position of the same vortices is randomized. Figures 3(a) 

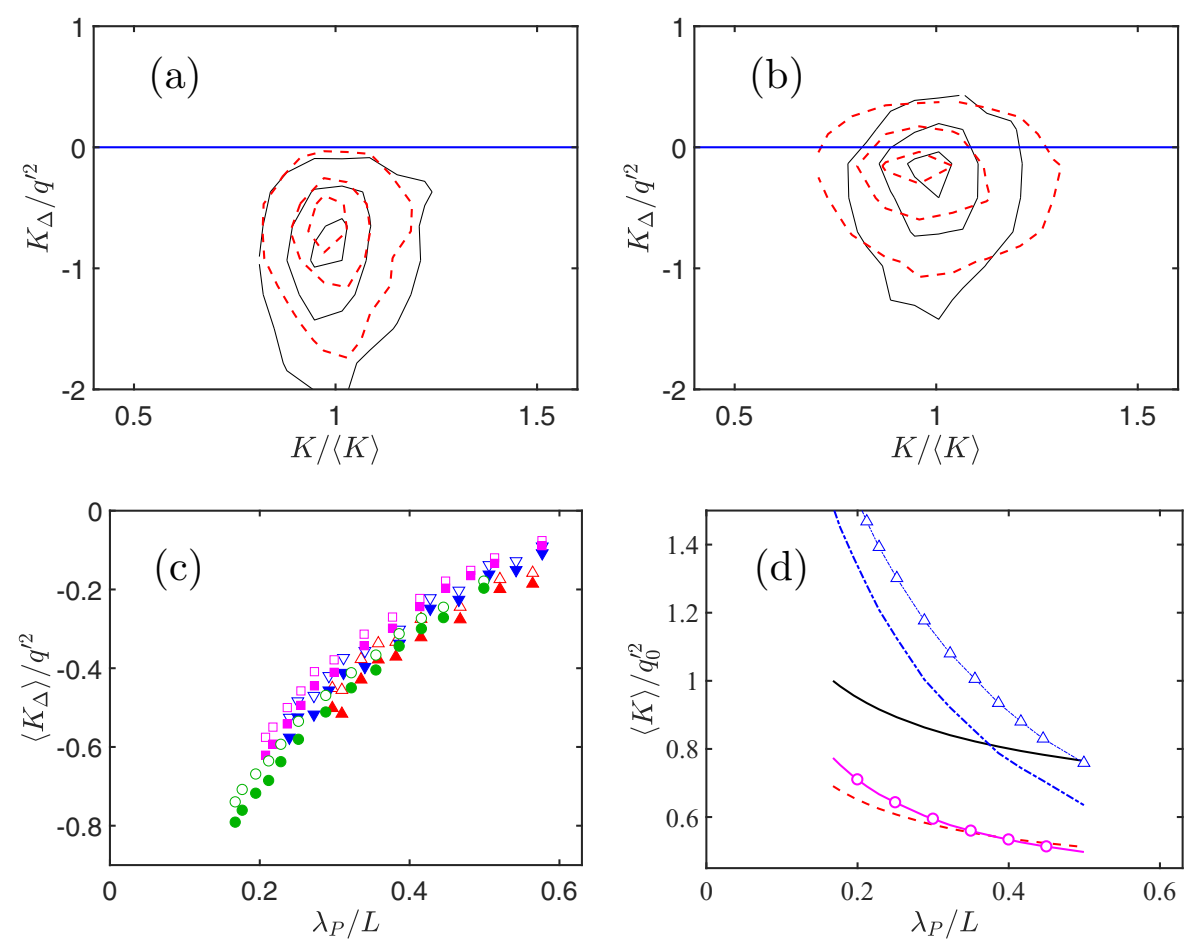

FIG. 3. (a) Joint probability density function of the kinetic energy difference $K_{\Delta}=K-K_{s}$ between the extended-vortex flows $K$ and the same flow in which the vortex positions are randomized $K_{s}$, i.e., T1024a at the time of Fig. 2(a). (b) Same as in (a) but at the time of Fig. 2(b). (c) Ensemble average of the randomizing energy versus $\lambda_{P}$. Symbols are as in Table I. In (a)-(c), open symbols and solid lines are the turbulence vortices and closed symbols and dashed lines are the point-vortex approximation. Contours in (a) and (b) contain 10\%, $50 \%$, and $90 \%$ of the probability. (d) Kinetic energy evolution for T1204a: - , total kinetic energy; - - - , energy from all vortices; - ०-, energy from large vortices; - - - - , energy from scrambled large vortices; and $-\Delta-$, self-energy approximation to the scrambled large vortices.

and 3(b) are joint probability density functions (PDFs) of the kinetic energy of the members of the ensemble,

$$
K=L^{-2} \int_{L^{2}}|\boldsymbol{u}|^{2} d^{2} \boldsymbol{x}
$$

versus the energy required for randomization $K_{\Delta}=K-K_{s}$. Particularly at the earlier evolution time in Fig. 3(a) [one of whose members is represented in Fig. 2(a)], the scrambling of the vortex position almost always leads to higher energies, although the effect weakens as the flow evolves towards fewer vortices in Figs. 3(b) and 2(b). Some caution is required in interpreting this result, because the vortices occasionally overlap after being rearranged, modifying the total enstrophy and the energy. The overlap is of the order of $7-10 \%$ of the vortex area and while the effect on the enstrophy is statistically neutral (not shown), that on the kinetic energy is not. Its magnitude is tested in Figs. 3(a) ad 3(b) by repeating the scrambling experiment after substituting each vortex by a compact point core with the same circulation, located at its center of gravity (see Appendix A). The overlap then decreases by a factor of 2-10 (see Appendix B), depending on the case, but the effect of the scrambling is still to increase the energy. Figure 3(c) summarizes the energy increase for all the experiments and evolution times. The best collapse is obtained by plotting it against $\lambda_{P} / L$ or, equivalently, against the number of vortices in the periodic domain. 
The kinetic energy of a sparse zero-circulation system of two-dimensional small vortices is [32]

$$
2 \pi K \approx\left(\sum_{i} \gamma_{i}^{2}\right) \log \left(\frac{\delta}{\ell}\right)+\sum_{i} \sum_{j \neq i} \gamma_{i} \gamma_{j} \log \left(\frac{r_{i j}}{\ell}\right)+\cdots,
$$

where $r_{i j}$ is the distance between vortices, $\ell$ is an arbitrary length scale, and $\delta$ is of the order of the radius of the vortex cores. The first term on the right-hand side of this equation is the self-energy and it is independent of the vortex position. It derives from the $1 / r$ velocity in the neighborhood of the cores and diverges as $\log (\delta)$. The second term in (5) is the interaction energy. It is independent of $\delta$ and only depends on the relative vortex position.

When (5) is modified to take into account the spatial periodicity of the computational box, the argument of the logarithms gains a Jacobian elliptic function [33], which does not change the shortrange interactions but introduces $\ell=L$ as a natural length scale. With this choice, the self-energy of the cores increases with decreasing core size and is 2-3 times larger for the point-vortex model than for the extended vortices of the original turbulence. This is the reason why the abscissas in Fig. 3 have to be scaled with the individual average for each system, but the figure shows that the interaction energy spent in randomization is the same for both flows, which is why their ordinates can be scaled with the same energy ( $q^{\prime 2}$ of the turbulent flow).

One of the most intriguing features of Fig. 3(c) is that the magnitude of the available interaction energy is initially of the same order as the total kinetic energy of the flow, but that it almost vanishes at the end of the simulations. This appears to contradict the result in Fig. 1(a) that the kinetic energy stays approximately constant during the decay and the implication is that most of the interaction energy is never expressed in the flow. The energy redistribution mechanism responsible for keeping vortices in a reduced-energy state is conservative, unrelated to dissipation, and the position of the vortices in a turbulent flow is always far from random.

Consider Fig. 3(d), which shows the evolution of several components of the energy of the flow as it decays. The black solid line is the total kinetic energy, which decays by about $20 \%$. The same is approximately true of the part of the kinetic energy contained in the large vortices, which closely tracks the total. The approximately $20-25 \%$ separating the two energies is presumably contained in the incoherent background. Including the vortices that we have classified as small (red dashed line) does not change the picture [4]. They actually subtract some energy from the flow during most of the evolution. The largest energy reservoir is in the set of randomized vortices (blue dash-dotted line), which is almost exclusively self-energy. The triangles in Fig. 3(d) are an approximation to the kinetic energy that essentially treats the first term in (5) as a sum of independent variables (see Appendix C). It represent the data well, especially taking into account that the turbulence vortices are far from circular and that the definition of $\delta=\left\langle(s / \pi)^{1 / 2}\right\rangle$ is ambiguous.

Figure 4(a) examines the energy distribution in more detail. The red solid lines are PDFs of the kinetic energy for the ensemble of turbulent flows, plotted at three evolution times that increase in the direction of the arrow and of the darker shades of color. In each case, the energy is normalized with the average energy of the ensemble of scrambled flows at the same time and the rightward drift of the PDF corresponds to the upward trend of the open symbols in Fig. 3(c). The black dashed lines in Fig. 4(a) are PDFs of the energy of the scrambled flows, scaled with their own mean. They change little with time, and we have seen that they mostly include the self-energy of the vortices. It could be expected that, since the vortex positions are randomized and since the velocity at each point of the flow is the sum of the contributions of the different vortices, the central-limit theorem would result in a Gaussian PDF, but [31] examined this limit and concluded that the $1 / r$ velocity distribution due to each core in a system of random compact vortices is marginal for the application of the central-limit theorem. Although the PDF of the resulting velocity would tend to Gaussian for a very large number of vortices, the rate of convergence is too slow to be useful in any practical sense. As a consequence, the distributions in Fig. 4(a) are skewed, as in [31]. Finally, the blue dash-dotted curves in Fig. 4(a) are the interaction energy, which has been isolated by subtracting the energy of two different randomizations of the same flow $K_{\Delta \Delta}=\left(K_{s 1}-K_{s 2}\right) / \sqrt{2}$. These distributions are 

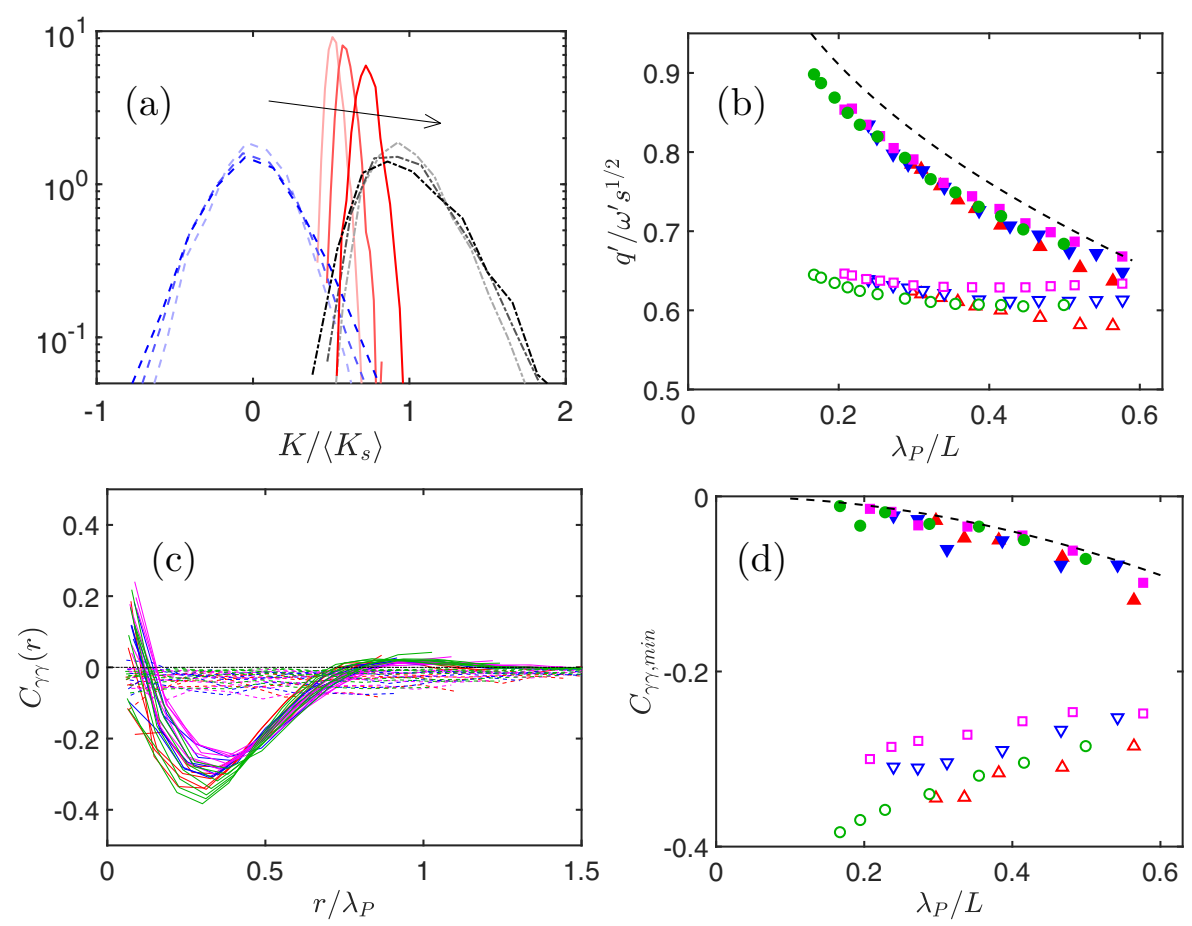

FIG. 4. (a) One-dimensional PDF of the kinetic energy of the reconstructed flows for T1024a: -, unscrambled vortices; - - - - , scrambled vortices; and - - - difference between two scramblings of the same flow. Time increases from light to dark, $\omega_{0}^{\prime} t=1.3,19.6,52.3$, and all energies are normalized with the mean scrambled energy at each time. (b) Normalized velocity intensity. Symbols are as in Table I. Open symbols are unscrambled flows; solid ones are scrambled. The dashed line is the logarithmic correction (6). (c) Correlation of the vortex circulation as a function of their distance for all cases and times. Colors are as in (b) and denotes unscrambled and -- - scrambled. (d) Minimum of the correlations in (c). Symbols are as in (b), but the dashed line is (8).

symmetric with zero mean, by construction, and, at least for the flows represented here, collapse well when scaled with the mean scrambled energy. Their standard deviation is of the order of 25-30\% of this mean, proving that there is enough energy in the vortex interactions to explain the energy required for scrambling.

It is significant that the distribution of the energy within the ensemble of real flows is much narrower than in the scrambled ones, implying again that turbulence only explores a small subset of the possible vortex distributions. Figure 4(b) shows the scaling of the kinetic energy with the enstrophy. Dimensionally, $q^{\prime} \sim \omega^{\prime}\langle s\rangle^{1 / 2}$, where $\langle s\rangle$ is the average vortex area, but there is a logarithmic correction (Appendix C), with the same origin as in Fig. 3(d),

$$
\frac{q^{\prime 2}}{\omega^{\prime 2}\langle s\rangle} \sim \log \left(\frac{\ell_{0}}{R}\right),
$$

where $\ell_{0}$ and $R$ are outer and inner limits, respectively, of the region over which the velocity induced by a vortex core decays as $1 / r$. The inner scale is always of the order of the vortex radius $R \sim\left\langle s^{1 / 2}\right\rangle$, but $\ell_{0}$ depends on the vortex distribution. For a random distribution of vortices in a box of side $L$ we can assume $\ell_{0} \approx L / 2$ and, since we saw in Fig. $1(\mathrm{~d})$ that $\left\langle s^{1 / 2}\right\rangle \approx \lambda_{P} / 4$, the right-hand side of (6) is approximately $\log \left(2 L / \lambda_{P}\right)$. The ratio $q^{\prime} / \omega^{\prime}\left\langle s^{1 / 2}\right\rangle$ is plotted in Fig. $4(\mathrm{~b})$, where the difference between the scrambled and unscrambled flows is equivalent to the one in Fig. 3(c). It is plotted against $\lambda_{P} / L$, and the logarithmic correction (6) is included as a dashed line. It describes the trend 
of the randomized flows well. Interestingly, the normalized energy of the original turbulent flows satisfies the uncorrected constant scaling much more closely than the scrambled ones, consistent with $\ell_{0} / R \approx 1.5-2$. This suggests that the turbulence vortices suppress each other's $1 / r$ velocity field beyond distances of the order of $d / \lambda_{P} \approx 0.3-0.4$, which is also the typical distance between nearest neighbors.

That neighboring vortices are not randomly distributed is confirmed by Fig. 4(c), which displays the correlation coefficient of vortex circulation as a function of distance

$$
C_{\gamma \gamma}(r)=\frac{\left\langle\gamma_{i} \gamma_{j}\right\rangle}{\left\langle\gamma_{i}^{2}\right\rangle},
$$

where $r=\left\langle r_{i j}\right\rangle$ and the average is taken over the ensemble and over all the $(i \neq j)$ combinations. Since, on average, there is the same number of vortices of each sign, one could expect this correlation to vanish. This is approximately true for the scrambled flows, plotted in the figure as dashed lines, but turbulent flows behave differently and the correlation reaches a negative minimum at $r \approx 0.33 \lambda_{P}$. This is close to the distance between neighboring cores [Fig. 1(d)] and implies that near neighbors tend to be of opposite sign. We noted when discussing (3) that the distribution of distances among neighboring vortices is consistent with a Poisson distribution and the same is found in [4], but when the vortices in that paper are grouped into closest pairs, the number of counterrotating dipoles is found to be approximately 50\% larger than that of corotating pairs. This effect disappears when the vortex position is randomized.

The minimum values of the correlations in Fig. 4(c) are collected in Fig. 4(d). Those from the turbulent flows increase slowly with increasing $\lambda_{P} / L$, but those from the scrambled ones do the opposite, and it is intriguing that they are always negative, even if they are independent of the distance $r$. This would seem to imply that some structure remains in the randomized flows, but the explanation is probably simpler and, as we will see below, significant. Consider a flow with $n_{v}$ identical vortices of balanced sign. Each vortex sees $n_{v} / 2$ vortices of opposite sign, for which $\gamma_{i} \gamma_{j}<0$, but only $n_{v} / 2-1$ vortices for which $\gamma_{i} \gamma_{j}>0$, discounting itself. Using (3), the resulting average is

$$
C_{\gamma \gamma} \approx-\frac{1}{n_{v}}=-0.25\left(\frac{\lambda_{P}}{L}\right)^{2} .
$$

This line has been added to Fig. 4(d) and represents the data well.

Screening of the far-field electrostatic potential by the redistribution of charges is well known in plasmas and electrolytes, where a charged particle induces a shell of charges of opposite sign that shelters its effect at long distance (see [34], Sec. 75). This sheltering reduces the total potential energy, and it was speculated in [29] that a similar effect could be present in turbulence. There is little empirical or experimental evidence either way, although $[35,36]$ observed that the absence of long-range pressure correlations in simulations of three-dimensional isotropic turbulence could be due to screening and generalized the argument to other three-dimensional flows. On the contrary, [31] found that a random vortex distribution describes well the second-order moments of decaying two-dimensional turbulence and interpreted this as evidence for the absence of screening. The reason for this discrepancy with the present results is not clear, but the two simulations are difficult to compare, because [31] uses fourth-order hyperviscosity as a dissipation model. Hyperviscous vortices are typically smaller than viscous ones and the area fraction covered by the vortices in that simulation is an order of magnitude smaller than in the present one. It is likely that $q^{\prime}$ in [31] is mostly self-energy.

On the other hand, electrostatic screening is quite different from vortex rearrangement, as already noted in [29]. The electrostatic Debye-Hückel screening length arises from a balance between the potential energy of the charge distribution and the kinetic energy of thermal agitation [34]. The rationale for vorticity screening would also be to minimize total energy, and it was already speculated in [4] that the predominance of dipoles in simulations could be an energy effect. However, 
although it is clear from (5) that tight pairs of cores of opposite sign decrease the interaction energy with respect to those of the same sign, this is due to their effect on the kinetic energy in the far field. There is no potential energy among vortices, and calculations of the evolution of point-vortex systems (see Appendix A) reveal no screening effect. Their correlation coefficient (7) remains equal to zero up to an inner limit determined by the temporal resolution of the numerical scheme, and the distribution of distances among close vortices is consistent with Poisson statistics, both for pairs of the same sign and for dipoles.

In fact, the evolution of inviscid point vortices and the decay of two-dimensional turbulence are fairly different problems, because, as mentioned in the Introduction, the former is a Hamiltonian system that conserves energy, momentum, and, by default, enstrophy, while the latter is dissipative. However, there is persuasive evidence that they are not completely unrelated and [8] showed that substituting points for the largest vortices in decaying turbulence reproduces well their motion for short times, while [17] showed that adding a simple merging rule for like-signed vortices, designed to conserve energy while dissipating enstrophy, extends the validity of the model to longer decays. On the other hand, if screening were just a local energy effect, it should be visible in the Hamiltonian simulations, which we have seen not to be the case. A fair amount of work has gone into the equilibrium solutions of sets of point vortices, from vortex crystals that are steady in some frame of reference [37] (see also Sec. VI below) to maximum-entropy distributions which are only statistically stationary [6,11]. These last two papers center on the statistical mechanics of sets of point vortices, in terms of an interaction energy measured with respect to the random distribution. They find that, when the interaction energy is positive, the two vortex signs separate into large coherent regions of opposite circulation, as in the negative temperature states in [10]. For negative energies, equivalent to positive temperatures, the two signs stay mixed. It follows from Figs. 3 and 4 that the latter is the regime of our decaying flows, except perhaps for distances of the order of the separation between immediate neighbors.

The closest equivalent to screening in the context of vortex flows is shear sheltering [38-40], in which a vortex approaching a strong vorticity interface deforms it in such a way as to cancel the long-range effect of the vortex. This has been invoked, for example, to explain the persistence of strong vortices in two-dimensional turbulence [39] and of regions of very different turbulence intensities in three-dimensional turbulence [40], but the effect is restricted to the interaction of vorticity structures of very different magnitude. Thus, while sheltering might be involved in the separation of vortex cores into large and small, it is unlikely to be the reason why the strong vortices rearrange themselves into low-energy configurations.

More promising is an extension of the argument leading to (8) and it may be relevant that, if this equation is extrapolated to $\lambda_{P} \approx L\left(n_{v} \approx 5\right)$, it approaches $C_{\gamma \gamma \text {, min }} \approx-0.25$, which is of the same order as the observed minima for the turbulence data. This, together with the observation that the negative correlations are restricted to distances of the order of the nearest vortex neighbors, suggests that the vortex circulation tends to be in balance over neighborhoods of $O\left(\lambda_{P}\right)$ in such a way that each vortex sees an excess of neighbors of the opposite sign. There are several ways in which this might be implemented. The simplest one is probably vortex amalgamation. When two point vortices are drawn together by a multivortex interaction, they tend to stay together for some time, among other reasons because separating them requires exchanging an energy that may not be locally available. This is true for point vortices of any sign, but the long-term evolution of pairs of extended patches depends on their relative sign. Corotating pairs tend to merge [41], so one of the partners disappears from the local count, while counterrotating dipoles are stable [7,42]. This would explain the prevalence of dipoles over corotating pairs and the local correlation minimum in turbulence simulations, as well as the absence of both effects in point-vortex systems.

Vortex amalgamation depends on the ratio of the vorticity to the strain that the partners induce on each other [43], which, for approximately similar vortices, only becomes relevant at distances of the order of the vortex radius [44]. This would be consistent with the observation in [4] that the distribution of vortices in decaying turbulence satisfies Poisson statistics except at distances of the order of $\lambda_{\omega}$, proportional to the vortex diameter. On the other hand, Fig. 4(c) suggests that the 

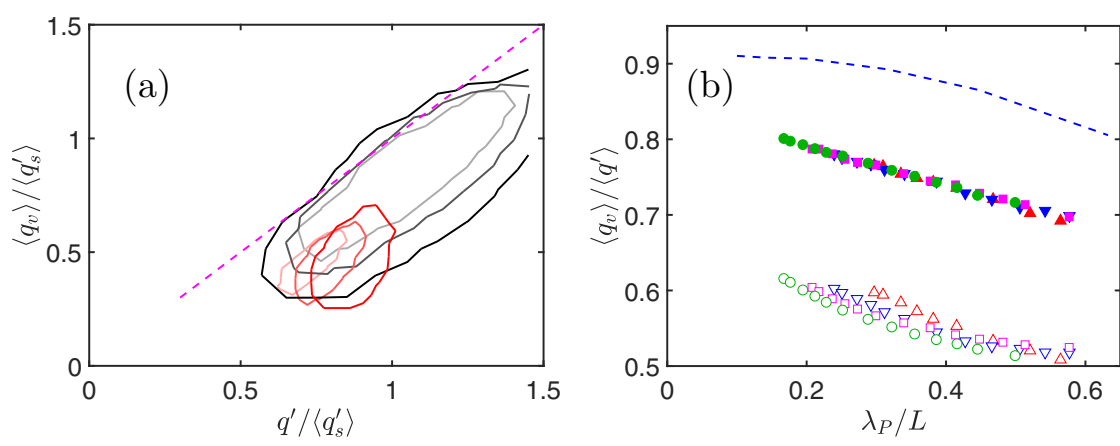

FIG. 5. (a) Joint PDF of the rms velocity and mean vortex mobility. Red lines are for large-vortex turbulence and black lines are for scrambled positions. Time increases from light to dark, $\omega_{0}^{\prime} t=1.3,19.6,52.3$, and all the energies are normalized with the mean scrambled energy at each time. Contours contain $99 \%$ of the probability mass. (b) Ratio between mobility and rms velocity for all cases. Symbols are as in Table I. Open symbols are unscrambled flows, solid ones are scrambled, and the dashed line is for the Hamiltonian evolution of sets of point vortices with an exponential distribution of circulation. The $\lambda_{P}$ for the point sets is defined using (3).

circulation is correlated over distances of the order of $\lambda_{P}$, which is of the order of the distance among vortices, but we saw in Fig. 1(d) that distance and diameter are proportional to each other for the large vortices. In fact, mutual shredding provides a plausible mechanism to enforce this proportionality [44]. Note that, in this interpretation, screening in turbulence would be closer to the Roche mechanism by which celestial bodies are destroyed by tidal forces in a strong gravitational field [45] than to the charge redistribution in plasmas.

\section{VORTEX MOBILITY}

The second interesting property of the system of large vortices is that they move more slowly than would be expected from the root-mean-square velocity of the flow [4]. In particular, if we define the mobility of a vortex core as

$$
q_{v}=s^{-1}\left|\int_{s} \boldsymbol{u} d^{2} \boldsymbol{x}\right|,
$$

where the integral is taken over the vortex support, it would appear that $\left\langle q_{v}\right\rangle$ should be approximately equal to $q^{\prime}$. Figure 5(a) shows that this is not the case. The black contours in the figure are joint probabilities of rms velocity versus mobility in randomized vortex systems. As could be expected, flows with lower kinetic energy also induce a lower mobility in their cores, but real turbulence, represented by the red contours, not only has a narrower distribution of energy than the scrambled flows, as in Fig. 4(a), but also lies at the lower edge of the range of mobilities. Considering that the vortices that have been used for the black contours are the same ones used for the red ones and that the contours enclose $99 \%$ of the probability mass in both cases, the vortex distributions in turbulence correspond to very improbable geometric arrangements. For example, the distance between two probability distributions $p_{1}(\boldsymbol{q})$ and $p_{2}(\boldsymbol{q})$, in a parameter space $\boldsymbol{q}$, can be quantified by the Hellinger norm [46], defined by

$$
H^{2}\left(p_{1}, p_{2}\right)=\frac{1}{2} \int\left(\sqrt{p_{1}}-\sqrt{p_{2}}\right)^{2} d \boldsymbol{q}
$$

which vanishes for $p_{1}=p_{2}$ and reaches its maximum $H=1$ for disjoint distributions. In the case of the scrambled and unscrambled distributions in Fig. 5(a), it varies from $H \approx 0.97$ at early times, when there are many vortices, to $H \approx 0.75$ at the end of the simulations. 
(a)

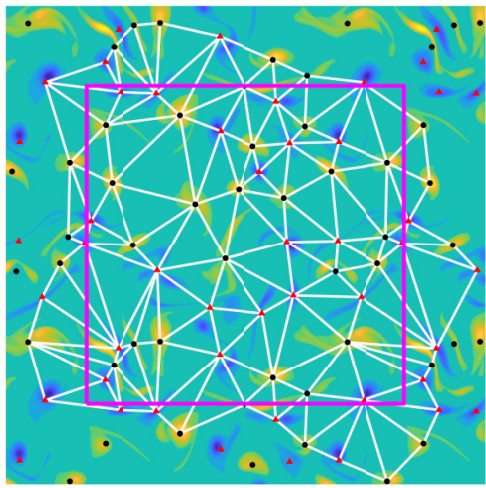

(b)

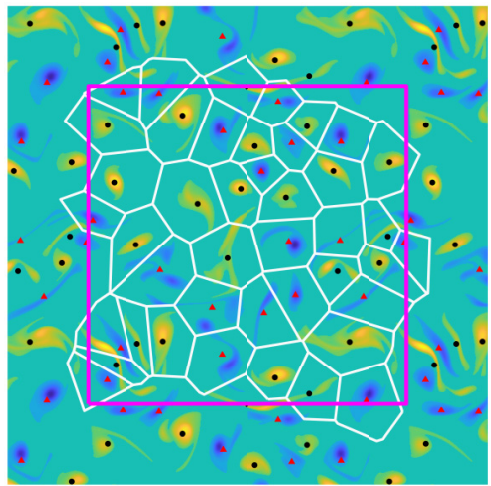

FIG. 6. (a) Delaunay triangulation of the lattice of vortex centers. Black dots are positive vortices; red dots are negative. The purple frame is the periodic computational box and only triangles containing a vertex within the box are plotted. For the case T512, $\omega_{0}^{\prime} t=2.8$ and $\lambda_{P} / L=0.30$. (b) Dual Voronoi tessellation of the same field.

Even if the scrambled flows in Fig. 5(a) tend to have $q_{v}<q^{\prime}$, probably because (9) acts as a filter that smooths the velocity fluctuations, Fig. 5(b) shows that the low mobility of the turbulence vortices goes beyond this effect. The closed symbols in this figure are the ratio between the mobility and the fluctuation intensity in scrambled flows. It is of the order of 0.75 . The open symbols are for turbulence and even if the vortices in both cases are the same, and therefore subject to similar filtering effects, the turbulent flows have smaller ratios, of the order of 0.55 . Both ratios decrease for a decreasing number of vortices, in what is probably another manifestation of the self-exclusion effect in (8). The dashed line in Fig. 5(b) is computed for Hamiltonian systems of point vortices, which, as we saw above, have very different dynamics from turbulence. In addition, their mobility is a true advection velocity, rather than an average such as (9). Even so, even if their mobility ratio is closer to unity than in the case of extended vortices, it also decreases with the number of vortices. The simplest explanation, as in (8), is that the self-induced velocity of each vortex is included in $q^{\prime}$ but not in $q_{v}$ and that this difference becomes more important as the number of vortices decreases.

A possibility that needs to be discounted is that the low mobility of the turbulence vortices may be due to geometric jamming, since we saw in Fig. 1(d) that the intervortex distance is of the same order as their diameter. This is not tested by the randomization of the vortex position in Fig. 5, because we saw above that this scrambling leads to some vortex overlap, which makes jamming partly irrelevant, but it can be tested by randomly flipping the sign of the vortices, without modifying their vorticity distribution or their shape. This operation respects the vortex geometry and would be as jammed as the original flow field, but the results (not shown) are indistinguishable from those for scrambled positions in Fig. 5(b).

\section{VORTEX LATTICE}

We next address what makes the distribution of vortices in turbulent flows atypical. We will focus on the organization of the centers of gravity of the vortices within their local neighborhood, since the two previous sections suggest that the vortex distribution over long distances is essentially random.

The conjecture at the end of Sec. III about the structure of the vortex neighborhood can be tested by analyzing the lattice formed by the vortex centers. Consider the example in Fig. 6, where the Delaunay triangulation and its associated Voronoi tessellation have been superimposed on the centers of gravity of the vortices within the periodic domain marked by the purple frame [47]. The Delaunay triangulation is the most regular triangulation of neighboring lattice points and 

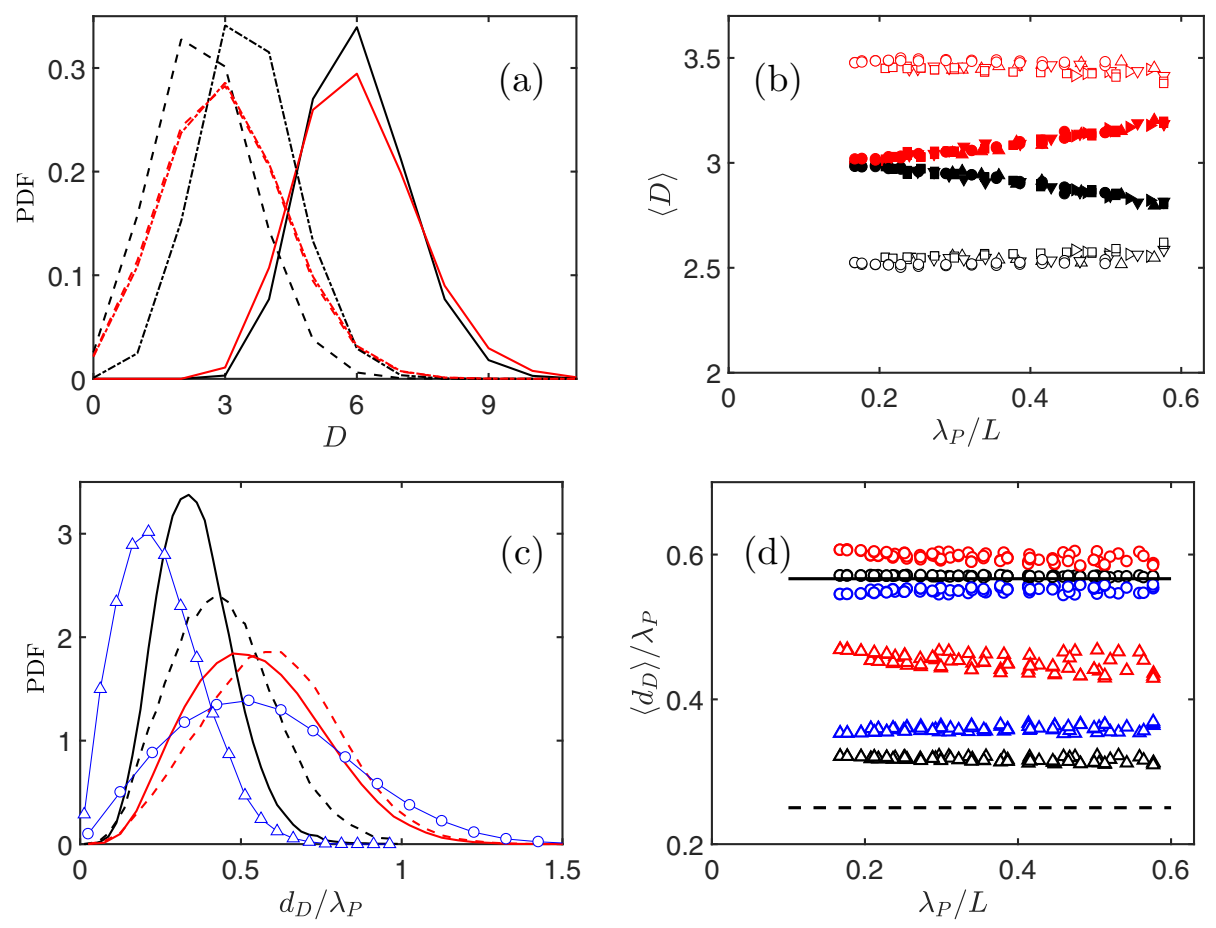

FIG. 7. Properties of Delaunay lattice neighbors. (a) PDF of the number of neighbors for T1024a, as in Fig. 2(a): - , all neighbors; - - , same circulation sign; and - - - - opposite sign. Red lines are for scrambled vortex positions. (b) Mean number of neighbors as a function of the number of vortices per field. Black symbols are corotating with the reference vortex. Red symbols are counterrotating. All cases are shown, with the symbols as in Table I. Open symbols are unscrambled flows; solid ones are scrambled circulations. (c) PDF of the distance between Delaunay neighbors for T2014a, as in Fig. 2(b). Red lines refer to all neighbors and black lines to the closest one in each neighborhood: - , counterrotating with the reference vortex; - - , corotating. Blue lines with symbols used scrambled vortex coordinates. Circles denote all neighbors and triangles the closest one. (d) Average distance from a vortex to its Delaunay neighbors. Circles denote all neighbors and triangles the closest one. Red symbols only include corotating vortices, blue are counterrotating, and black include both signs. The two horizontal lines are for scrambled coordinates: - , all neighbors; - - - , the closest one.

defines lattice neighborhoods. The Voronoi tessellation is formed by the sets of flow points which are closest to each vortex center and defines flow neighborhoods.

The lattice neighborhood of a vortex center can be defined as the set of centers connected to it by a Delaunay edge, and the conjecture in Sec. III can be expressed as that the lattice neighborhood of a vortex contains, on average, fewer vortices corotating with it than counterrotating ones. This would automatically be true for any reasonably homogeneous lattice by the argument in (8), and a second question is whether the observed effect is stronger than could be expected from a random point set. This is tested in Fig. 7(a), which shows the probability distribution of the degree, $D$ (number of neighbors connected to a given vortex), as well as of the number, $D_{+}$and $D_{-}$, of corotating and counterrotating neighbors, respectively. It is known that $\langle D\rangle=6$ for any planar triangulation [47]. This is a topological property, independent of flow structure, but it is clear from Fig. 7(a) that $\left\langle D_{-}\right\rangle>\left\langle D_{+}\right\rangle$. The average values of these partial degrees are given in Fig. 7(b). The open symbols are $\left\langle D_{+}\right\rangle$and $\left\langle D_{-}\right\rangle$; they depend little on the particular case or on the evolution time and reflect the average loss of one corotating vortex from each neighborhood. To test whether this loss is specific to turbulence, the triangulation is repeated after randomizing the circulation of the vortices. The 
results are included as closed symbols in Fig. 7(b). They show no difference between the two vortex signs at short evolution times, when the flow has many vortices, and drift towards the unscrambled results when the number of vortices decreases at later times. The result of randomizing the vortex position instead of the circulation is essentially the same. The origin and magnitude of this drift is the same as in Fig. 4(d) and in (8).

Figures 7(c) and 7(d) display the PDF and mean value of the distance from a vortex to its Delaunay neighbors, $d_{D}$, separated into corotating and counterrotating ones. The red lines in Fig. 7(c) and the circles in Fig. 7(d) refer to all the neighbors, while the black lines and the triangles refer to the closest one. In both cases they are compared with the result from fields in which the vortex positions are scrambled. The results depend relatively little on the evolution time or on the number of vortices. In all cases, the corotating neighbors are farther from the reference vortex than the counterrotating ones, in agreement with the tidal capture model discussed in Sec. III. This effect is weak in the case of all neighbors, $\left\langle d_{D}\right\rangle / \lambda \approx 0.59$ and 0.55 , respectively, compared to 0.57 in the randomized case, but much clearer in the case of the closest neighbor, $\left\langle d_{D}\right\rangle / \lambda \approx 0.44,0.36$, and 0.25 , respectively. Also striking is that the turbulence PDFs are narrower than the randomized ones, reflecting a more rigid geometry that could be interpreted as supporting the description of the lattice as a crystal. The main difference between turbulence and the randomized field is at short distances, where the vortices do not approach each other beyond $d \approx 0.25 \lambda_{P}$, of the order of their diameter [Fig. 1(d)]. This is again consistent with the tidal model, or even with simple geometric exclusion, and agrees with the result in [4] for nearest neighbors.

Figure 8 presents two more indicators of the tendency of turbulent flows to be more organized than random point sets. Figure 8(a) present PDFs of the diameter $s_{\text {Vor }}^{1 / 2}$ of the individual polygons in Voronoi tessellations, as in Fig. 6(b). The distribution depends very little on the evolution time and even on the flow parameters and is shown in the figure averaged over the whole flow decay. It follows from the area-covering property of tessellations and from (3) that $\left\langle s_{\text {Vor }}\right\rangle=\lambda_{P}^{2} / 4$, or $\left\langle s_{\text {Vor }}^{1 / 2}\right\rangle / \lambda_{P} \approx 0.5$. This is true for the centers of gravity of turbulence and for random point sets, but the standard deviation of the turbulence distributions $\left(0.08 \lambda_{P}\right)$ is much narrower than for random sets $\left(0.125 \lambda_{P}\right)$.

The organization of the vortices in the crown that forms the lattice neighborhood of a given vortex can be characterized by arranging them counterclockwise and measuring the angle $\alpha$ among the consecutive Delaunay edges joining them with the central core [see the sketch in Fig. 8(b)]. Since the average number of lattice neighbors is $\langle D\rangle=6$, one could expect $\langle\alpha\rangle=2 \pi /\langle D\rangle=\pi / 3$. Empirically, $\langle\alpha\rangle / \pi=0.33(1 \pm 0.5)$, which is essentially the same as for randomized vortex positions. The situation is different for corotating or counterrotating neighbors, for which $\left\langle\alpha_{ \pm}\right\rangle / \pi \approx 2 /\left\langle D_{ \pm}\right\rangle$ also holds, but the standard deviation $\left\langle\alpha_{ \pm}^{2}\right\rangle^{1 / 2} /\left\langle\alpha_{ \pm}\right\rangle \approx 0.6$ is higher than for the overall angles and lower than for randomized vortex positions or circulations $\left\langle\alpha_{ \pm}^{2}\right\rangle^{1 / 2} /\left\langle\alpha_{ \pm}\right\rangle \approx 0.7$. In fact, Fig. 8(c) shows that the distribution of the angular distance among corotating or counterrotating vortices is not just a shifted version of the PDF for all the vortices. Some of the Delaunay neighbors that corotate (or counterrotate) with respect to their reference vortex are immediate angular neighbors among themselves and the modal value of the angle distributions is close to the general mode. Their higher mean value is due to a tail of larger angles, required for the total to add to $2 \pi$. For example, the corotating vortices include a substantial spike at $\alpha_{+}=2 \pi$, corresponding to crowns with a single corotating partner.

The sign of the circulation of the vortices within each Delaunay crown is slightly more mixed than would correspond to a random distribution. Figure 8(d) shows the PDF of the length of the segments with similar sign around the crown. For example, the sketch in Fig. 8(b) is maximally mixed, i.e., its highest azimuthal persistence is one, because there are no contiguous vortices of the same sign. A minimally mixed crown for a set of balanced vortices with the average $D=6$ would have two segments of length 3. The dashed lines in Fig. 8(d) show that a set of random points mostly has contiguous segments of length 1 or 2 , with a relatively fat tail extending to full crowns of a single sign. The solid lines in the figure show that the turbulence PDF is slightly more peaked at unity than the random ones, with an average persistence of 1.7. It describes a fairly inhomogeneous crown 


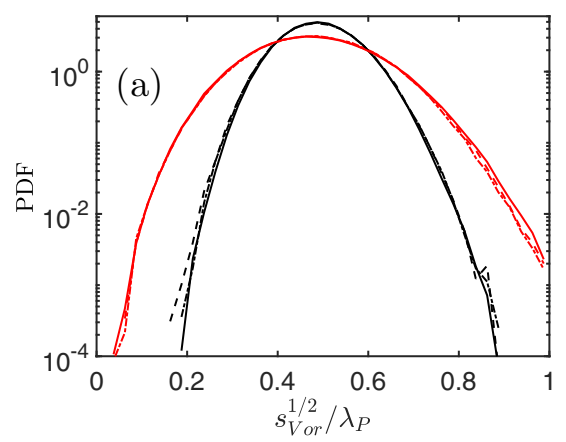

(b)
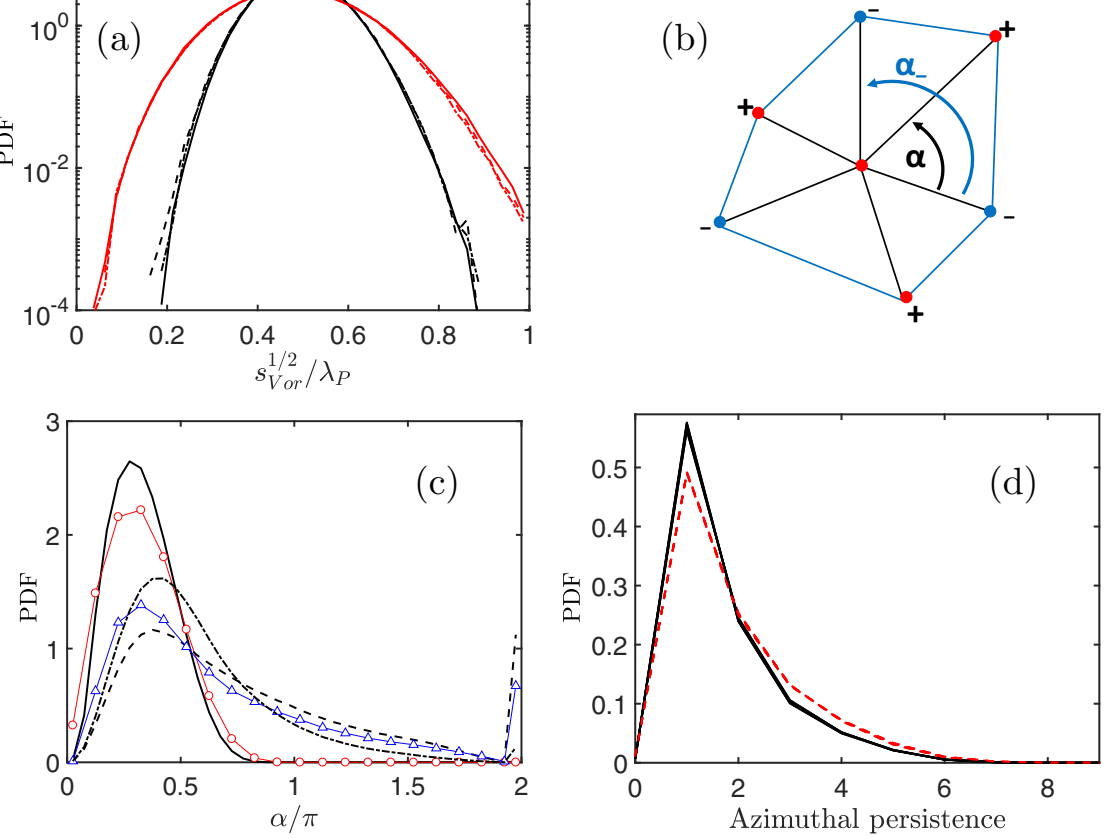

FIG. 8. (a) Probability density function of the diameter of the Voronoi tessellae:- - - - , T768; - - - , T1024; and —, T1204a. Black lines are turbulence and red lines are randomized vortex positions. Each PDF is the average of all simulation times. (b) Sketch of the definition of the angular distance between lattice neighbors $\alpha$ and between neighbors of a given kind $\alpha_{ \pm}$. (c) PDF of the angular distance among the lattice neighbors to a given vortex: - , all neighbors; - - , corotating with the central vortex; - - - - , counterrotating; $-\circ-$, all neighbors, with randomized coordinates; and $-\Delta-$, corotating or counterrotating neighbors, with randomized circulation. The case T1024a is shown, averaged over all times. (d) PDF of the length of the segments of mutually corotating vortices in the crowns of immediate neighbors to a given vortex: - , turbulence; - - - , randomized circulation. The figure includes all the cases in Table I, each one averaged over time, but the results are indistinguishable at the scale of the plot.

with fewer than two consecutive vortices sharing the same sign, although with a slight tendency towards isolated corotating vortices. The latter is consistent with the already discussed tendency for corotating vortices to avoid each other.

\section{CRYSTALS}

Before moving to our final attempt to extract ordered structures from the turbulent flow fields, it is useful to explore whether what we have found up to now can be interpreted as an approximation to stationary crystalline vortex lattices. Two-dimensional vortex crystals are well known [37]. Some of them are stable and form spontaneously in experiments. Forced two-dimensional turbulence is known to settle to stationary patterns which are partly determined by the forcing and by the boundary conditions [48-50], although many of the examples do not refer to the Navier-Stokes equations but to systems such as Bose-Einstein condensates, magnetized plasmas, or superfluids, whose dissipation mechanisms are not exactly those of Navier-Stokes fluids. Beautiful equilibrium vortex polygons have been observed in the polar regions of planetary atmospheres [51].

Most of these examples are regular arrangements of vortices of a single sign in a background of opposite-sign vorticity, but mixed-sign vortex systems with zero overall circulation also exist. The von Kármán vortex street is probably the best known and can be generalized to a doubly periodic 
(a)

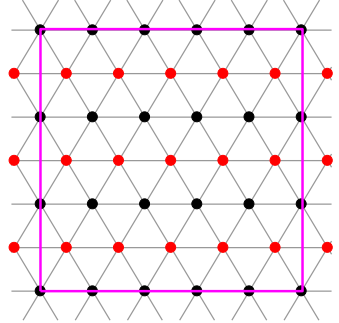

(b)

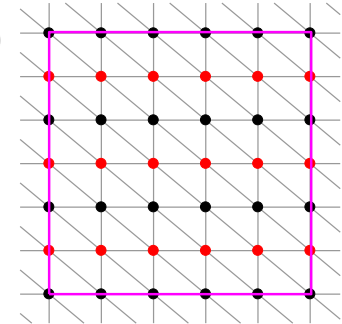

(c)

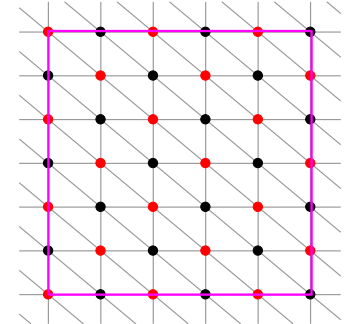

FIG. 9. (a) Approximately hexagonal lattice of staggered von Kármán vortex streets. Red dots have positive circulation and blue ones are negative. (b) Rectangular lattice of aligned streets. (c) Rectangular checkerboard lattice. All these lattices are stationary equilibrium solutions.

stationary lattice. Consider the two examples in Figs. 9(a) and 9(b). There are two equilibrium configurations of a double street of point vortices, both of which move with a constant velocity (see [52], Sec. 156). In the first one, two lines of vortices of opposite sign are staggered, and stacking an infinite number of these staggered streets, as in Fig. 9(a), results in an approximately hexagonal lattice. In the aligned equilibrium configuration of two vortex rows, the vortices are vertically aligned with respect to each other, instead of staggered, and they generalize to the cubic lattice in Fig. 9(b).

While the staggered and aligned von Kármán streets are the only known equilibrium solutions for two lines of vortices of zero total circulation, there are many more doubly infinite equilibrium lattices. Enumerating and analyzing them is beyond the scope of this paper, but an example is given in Fig. 9(c). Note that the three lattices in Fig. 9 are rotated versions of each other for particular aspect ratios of order unity, but they are generally distinct and they share a structure of alternating layers of vorticity that induce high-velocity streams which could be used as a model for the streams observed in Figs. 2(c) and 2(d).

The interaction energy of these lattices depends on the aspect ratio, defined as $\eta=\Delta y / \Delta x$, where $\Delta x$ is the pitch within each row and $\Delta y$ is the distance between rows. This energy can be computed analytically in some cases [53], but it is most easily determined by approximating the lattice by a system of compact cores, as in Sec. III, and subtracting the self-energy estimated in Appendix C. This is done in Fig. 10(a), where energies are represented normalized per core, which shows that the regular lattices are low-energy states with respect to randomized vortex distributions. The dashed line is the normalized interaction energy for truly random vortex-core systems, computed in the
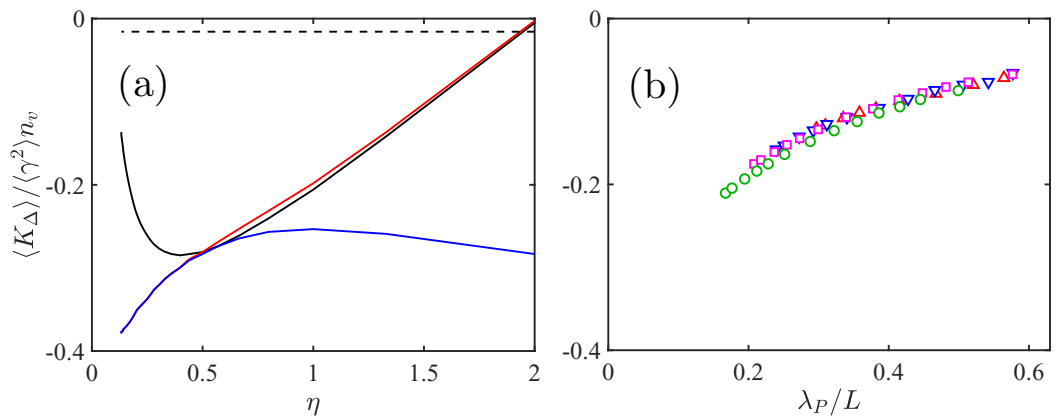

FIG. 10. (a) Normalized interaction energy for the three lattices in Fig. 9 versus the aspect ratio. Black denotes a staggered von Kármán lattice, red an aligned lattice, and blue a checkerboard lattice. The dashed line is a random superposition of between $n_{v}=2$ and 100 Gaussian cores in the computational box. (b) Normalized interaction energy for the point-vortex approximation to the turbulence simulations. Symbols are as in Table I. 
same way, and its small difference from zero is a measure of the accuracy of the estimates in Appendix C.

Figure 10(b) contains the energy for the point-vortex approximation to the turbulence simulations in Table I, normalized as in Fig. 10(a), and shows that turbulence goes a good part of the way to minimizing its energy, reaching values comparable to those of infinite regular lattices. It would be nice to relate this low energy to the stability of the different configurations, but energy is not a good indicator of stability. The staggered von Kármán double street is unstable for all aspect ratios except $\eta_{c} \approx 0.28055$, at which it is neutrally stable to linear perturbations [52,54], but its energy increases monotonically with increasing $\eta$, without any special behavior at $\eta_{c}$. The lattices in Fig. 9 have been drawn with an aspect ratio of order $\eta=\frac{1}{2}$, suggested by isotropy. Figure 10(a) shows that this is close to the minimum energy configuration for the staggered von Kármán lattice in Fig. 9(a), but this should not be taken as a stability criterion. It is known that an equilateral triangular lattice is the only stable case for vortex lattices of a single sign [55], but the stability of the mixed cases in Fig. 9 is unknown.

We are now in a position to discuss in which sense, if any, the statistics of turbulence approximate a lattice such as those in Fig. 9. Properties such as a uniform Voronoi area [Fig. 8(a)] or a constant Delaunay degree [Fig. 7(a)] are trivially satisfied by most regular lattices. The angle distribution in Fig. 8(c) is not different enough from those of randomized vortices to single out a particular lattice, but its narrow distribution around $\pi / 3$ rules out the symmetries in Figs. 9(b) and 9(c), which are closer to a bimodal distribution of angles with peaks around $\pi / 4$ and $\pi / 2$. The azimuthal persistence in Fig. 8(d), with approximately twice as many isolated vortices of one sign as the number of corotating pairs, is consistent with any of the layered lattices in Fig. 9, but not with isolated vortices surrounded by crowns of opposite circulation.

It is interesting that, although we have discussed the imbalance between corotating and counterrotating neighbors as indications of out-of-equilibrium behavior, all the equilibrium lattices in Fig. 9 have twice as many counterrotating Delaunay neighbors as corotating ones. This is another consequence of the organization into layers, but is probably not enough to explain the results in Fig. 7. For one thing, the observed imbalance in the number of neighbors of each class [ 3.5 to 2.5 in Fig. 7(b)] is not close enough to 2 to unambiguously define a regular layered lattice. The ratio between the average distance of the Delaunay neighbors to the distance to the closest one [Fig. 7(d)] is more informative. This ratio is fairly close to unity for all the lattices in Fig. 9. Even for the square lattices in Figs. 9(b) and 9(c), it is about $d_{a v} / d_{\min } \approx 1.14$. The average-to-minimum ratio for vortices of all classes in Fig. 7(d) is approximately 1.8, much closer to the random value 2.3 than to any regular grid. The set of counterrotating vortices is intermediate between random and regular lattices $d_{a v} / d_{\min } \approx 1.54$, but the most regular distribution of distances is for the set of vortices corotating with the center, for which $d_{a v} / d_{\text {min }} \approx 1.34$. Moreover, Fig. $7(\mathrm{~d})$ shows that the mean distance to the central vortex is very similar for the three vortex classes, but that, in agreement with our previous analysis, it is the tight pairs of corotating vortices that are missing.

In summary, although the statistics presented up to now for the turbulence lattice are consistent with local double layers of counterrotating vortices, they probably cannot be used by themselves as indicators of an ordered lattice over long distances.

\section{CRYSTALLOGRAPHY}

We finally investigate whether the global vortex organization can be characterized using Fourier techniques inspired by x-ray crystallography. At first sight, this seems difficult. Fourier analysis of turbulence is similar to powder crystallography, in which diffraction takes place over disparate small crystals with random orientations and whose typical outcome is only a set of length scales [56]. There are two sources of randomness in the Fourier analysis of turbulent flows. The first one is that individual fields have random orientations, as in powders. The second is that the Fourier transform is an integral over the whole computational box and, since we have seen that nontrivial vortex arrangements probably only extend to local neighborhoods, the transform also mixes neighborhoods 
with random orientations and positions. The randomization due to the orientation of individual fields is relatively easy to compensate, because we have access to the spectrum of each field. The one due to the global transform requires substituting local expansions for global ones.

In view of the difficulty of identifying global symmetries, we center this section on the simpler problem of determining whether some specific symmetry applies, on average, to local neighborhoods of individual vortices. We know from previous sections that the mean distance between vortices is of the order of $\lambda_{P}$ and we define neighborhoods as disks whose radius is some low multiple $R_{P}=\mu \lambda_{P}$. Consider the point-vortex representation of the flow as a set of vortex centers located at $\boldsymbol{x}_{j}$, with circulations $\gamma_{j}$. To study the neighborhood of the $i$ th vortex, define local polar coordinates $\boldsymbol{x}_{j}-\boldsymbol{x}_{i} \rightarrow\left(r_{i j}, \theta_{i j}\right)$ and construct

$$
\hat{\Gamma}_{n}^{[i]}=\sum_{j} \gamma_{i j} \exp \left(-i n \theta_{i j}\right)
$$

where $\gamma_{i j}=\gamma_{i} \gamma_{j} /\left|\gamma_{i}\right|$ and the sum extends over the vortex centers satisfying $r_{i j} \leqslant R_{P}$. The effect of the sign factor $(\gamma /|\gamma|)$ in $\gamma_{i j}$ is to classify vortices as co- or counterrotating with the reference one $\boldsymbol{x}_{i}$ rather than as positive or negative. Equation (11) is essentially the angular Fourier transform of the projection on the unit circle of the locations of the vortices contained in a neighborhood disk and the complex $\hat{\Gamma}_{n}^{[i]}$ can be written as $\left|\hat{\Gamma}_{n}^{[i]}\right| \exp \left(i \psi_{n}^{[i]}\right)$. To overcome the problem of random orientations, we rotate the position of the vortices in each individual neighborhood so that some specified harmonic $n_{0}$ is made real and positive,

$$
\left(\tilde{r}_{i j}, \tilde{\theta}_{i j}\right) \rightarrow\left(r_{i j}, \theta_{i j}+\frac{\psi_{n_{0}}^{[i]}}{n_{0}}\right)
$$

thus rotating other harmonics in (11) to $\hat{\Gamma}_{n}^{[i]} \exp \left(-i n \psi_{n_{0}}^{[i]} / n_{0}\right)$ and $\hat{\Gamma}_{n_{0}}^{[i]}$ to $\left|\hat{\Gamma}_{n_{0}}^{[i]}\right|$. Intuitively, this rotation aligns the average position of the cloud of corotating (counterrotating) vortices to preferentially lie in one of the positive (negative) lobes of $\cos n_{0} \theta_{i j}$ and the resulting ensemble average is a conditional average of the vortex lattice in these aligned coordinates.

An example is Fig. 11(a), which presents results for an early time in the evolution of T1024a, oriented to $n_{0}=1$, and compares it to a similar flow in which the vortex positions have been randomized [Fig. 11(b)]. These figures are signed PDFs in which each vortex is counted as \pm 1 according to whether it co- or counterrotates with the central vortex of the neighborhood. Statistics are compiled over 2000 flow fields, which involve approximately $4 \times 10^{6}$ vortices in $2 \times 10^{5}$ neighborhoods. The result is a relatively compact concentration of counterrotating vortices to the left of Fig. 11(a), $x \approx-0.4 \lambda_{P}$, and a more diffuse distribution of corotating ones to the right. The randomized flow in Fig. 11(b) does not show the asymmetry between co- and counterrotating vortices. Its PDF simply reflects the structure of the orienting cosine in (11). Figure 11(a) is essentially a directionally unfolded version of the circulation correlation in Fig. 4(c), but a better way to render it is probably Fig. 11(c), where the turbulence and randomized distributions have been subtracted. This representation is less affected by artifacts due to the orienting cosine and becomes more informative when applied to fields oriented to some of the higher harmonics, as in Figs. 11(d)-11(f). Missing from all the maps in Fig. 11 is the central reference vortex, corotating with itself by default, which has been added as a disk at the origin with the radius implied by the average vortex size in Fig. 1(d). The compact counterrotating cloud present in all figures near the center is the screening vortex found in the correlations in Fig. 4(c). The compact dipole formed by these two vortices appears to be the statistically preferred state of the vortices in turbulence, as already seen in Secs. III and V and [4].

The more extended vortex clouds farther from the center, with separations of the order of $1.5 \lambda_{P}$, are too far to be considered part of the local neighborhood [see Fig. 7(c)] and belong to the category of the next-closest vortex. They are of the size of the kinetic-energy streams in Figs. 2(c) and 2(d). An important parameter of the analysis is the multiple $\mu \lambda_{P}$ that determines how many vortices are considered to be part of the local neighborhood disk. In general, larger neighborhoods result in a 
(a)

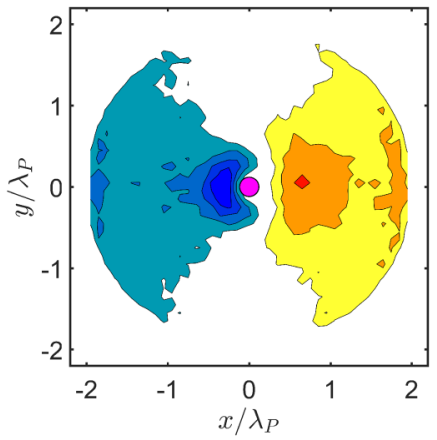

(d)

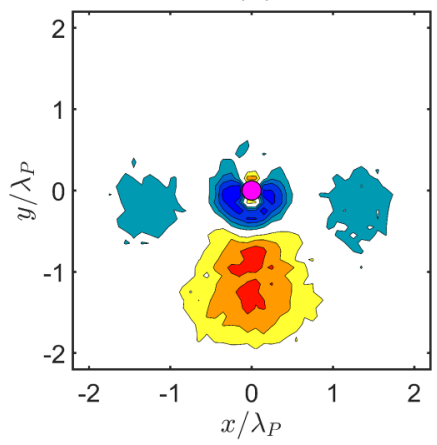

(b)

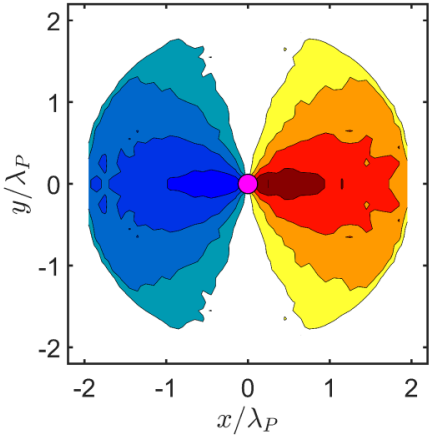

(e)

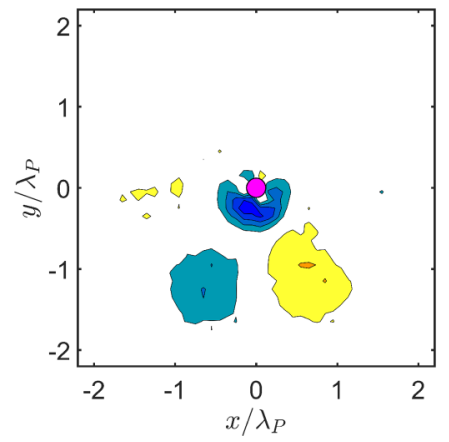

(c)

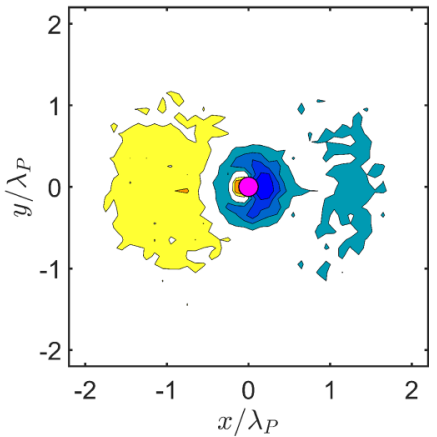

(f)

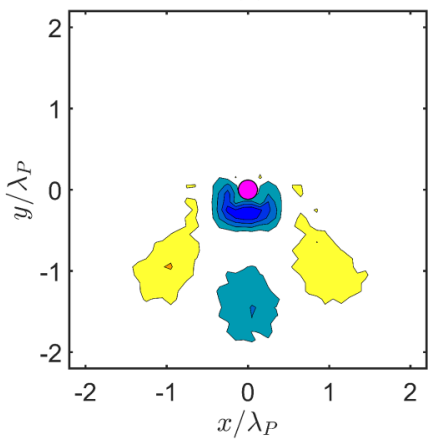

FIG. 11. Two-dimensional distribution of vortex positions in oriented flow neighborhoods, normalized to a unit maximum absolute value, for T1024a, as in Fig. 2, with $\mu=2$ : (a) turbulence, (b) randomized positions, and (c)-(f) turbulence minus randomized positions for (a)-(c) $n_{0}=1$, (d) $n_{0}=2$, (e) $n_{0}=3$, and (f) $n_{0}=4$. Contours are \pm [0.2:0.2:1]. Red contours are for corotating vortices and blue are counterrotating. All figures lack the central reference vortex, located at $x=y=0$, that is indicated by the purple circle whose radius corresponds to the average vortex size in Fig. 1(d).

clearer asymmetry, although the signal deteriorates when $R_{P} \gtrsim L / 2$ and the conditioning interacts with the periodicity of the numerics, but the outer vortex clouds change little as long as they fit within the analysis disk.

It is probably unwise to read too much into conditional averages, which always retain some influence from the conditioning. The underlying symmetry in Fig. 11 is dictated by the harmonic used in (12). However, the asymmetry between co- and counterrotating vortices in Figs. 11(a) and 11(c)-11(f) is a flow property, as shown by the comparison with Fig. 11(b). So is the separation among the different vortex clouds in the figures, and it is tempting to see in Fig. 11 fragments of the regular grids in Fig. 9. Note that the distances involved are rather large. We saw in Sec. II that a disk of radius $\lambda_{P}$ contains approximately 15 vortices. The analysis disk used in Fig. 11, with $\mu=2$, therefore represents the organization of about 60 vortices.

\section{CONCLUSION}

The results in this paper describe how decaying two-dimensional turbulence spontaneously organizes into a low-energy lattice of large vortices that is substantially more ordered than a random vortex distribution and evolves more slowly than other parts of the flow, but which is neither fully ordered over long distances nor stationary. It contains most of the kinetic energy of the flow and 
could perhaps be described as a vortex liquid rather than a gas or a crystalline solid. We may call it a stochastic crystal.

We have shown that what could be called the latent heat of randomization is not an issue in turbulence decay. The vortices in the flow never explore the randomized states and they approximately conserve energy by staying within a very restricted fraction of the configuration space of vortex positions. If we consider the process of vortex amalgamation during decay as a late stage of the condensation of the flow vorticity into compact cores, vortex formation and order generation are parts of the same process.

We have seen that maintaining a low relative energy of the vortex lattice requires mutual screening of the far field of the vortex-induced velocity, but that this is not implemented by attracting vortices of opposite sign to surround each other, as in electrostatics, but by preferentially absorbing vortices of the same sign, in a process akin to tidal disruption in strong gravitational fields.

The resulting vortex lattices are not organized enough to be considered equilibrium solutions, but they are shown by various geometric analyses to locally approximate double vortex layers, which move slowly and could perhaps be related to fixed points of the dynamical system representing the flow. However, the equilibrium is constantly disrupted in decaying turbulence by the capture process mentioned above, which ensures that the neighborhood of each vortex contains on average one more counterrotating vortex than corotating ones.

One of the referees called attention to the possible dependence of the above results on the initial conditions. This is an important issue in transient or decaying flows. It is known, for example, that the long-term behavior of the large scales in decaying three-dimensional turbulence depends on the small-wave-number slope of the initial energy spectrum, $E_{q q} \sim k^{\zeta}$, and that $\zeta=2$ and 4 are distinguished values that tend to persist during the decay (see [35] and references therein). This persistence is not preserved in two dimensions, because the inverse cascade continuously pumps energy into the large scales, where it condenses and destroys any preexisting spectral structure [25]. All our simulations use $\zeta=-1 / 2$ [4], but the shorter spectral peak of case T1024a was specifically introduced to explore the effect of different initial spectra. A few cursory experiments were also run with $\zeta=-2$ to $\zeta=4$ for the same purpose. The main effect of $\zeta$ is to change the rate at which the large scales condense, which also modifies the balance between disordered small vortices and ordered large ones. Very peaked spectra $(\zeta>0)$ introduce an initial family of vortices of approximately uniform size, which does not completely disappear by the time that the rest of the energy has condensed. Tests like the distribution of Voronoi areas in Fig. 8(a) suggest that the large vortices in these flows are even more organized than in the cases discussed in the present paper. For an initially uniform enstrophy spectrum $(\zeta=-2)$, the flow is condensed from the beginning and vortex families do not have time to form. The elucidation of the full range of possible behaviors would unfortunately extend this paper beyond any reasonable length and has not been attempted. The present results should be seen as evidence of the spontaneous segregation of a relatively organized subset of large vortices from an initial condition in which vortices have widely scattered (power-law) sizes and circulations.

Further investigation is needed to determine whether any of the mechanisms described here can be generalized to three-dimensional flows.

\section{ACKNOWLEDGMENT}

This work was supported by the European Research Council under the Coturb Grant No. ERC2014.AdG-669505.

\section{APPENDIX A: POINT-VORTEX SIMULATIONS}

There are two compact vortex approximations used for comparison in the text. This Appendix describes them and their numerical issues. 


\section{Gaussian cores}

The first approximation, used in Secs. III-VI, substitutes the precomputed turbulence vortices by Gaussian cores,

$$
\omega_{g}(\boldsymbol{x})=\left(\frac{\gamma}{\pi R^{2}}\right) \exp \left(-\frac{\left|\boldsymbol{x}-\boldsymbol{x}_{\mathrm{cog}}\right|^{2}}{R^{2}}\right),
$$

where $\gamma$ is the circulation of the turbulence vortex being approximated and the center of gravity is defined over the vortex support $s$ as

$$
\boldsymbol{x}_{\mathrm{cog}}=s^{-1} \int_{s} \boldsymbol{x} \omega(\boldsymbol{x}) d^{2} \boldsymbol{x}
$$

The core radius is arbitrarily set to $R / L=10^{-2}$, except for some convergence tests at $R / L=2.5 \times$ $10^{-3}$, and the core area, when needed, is defined as $s_{g}=\pi R^{2}$.

There is no dynamics in this operation, which represents an existing flow field. The cores are added to an empty $1024^{2}$ numerical grid, a uniform background vorticity is added to zero the overall circulation, and flow quantities of this synthetic field are computed in the usual manner.

The kinetic energy of the regular lattices in Sec. VI are also computed using this procedure.

\section{Hamiltonian point vortices}

In contrast to the postprocessing operation just described, some simulations were run for Hamiltonian systems of point vortices. Defining a complex variable $z=x+i y$, each vortex satisfies

$$
d_{t} \bar{z}_{j}=\bar{w}_{j}=u_{j}-i v_{j}=\sum_{k \neq j} \frac{\gamma_{k}}{2 \pi i\left(z_{j}-z_{k}\right)},
$$

where $\gamma_{k}$ is the circulation of the individual vortices and the overline denotes complex conjugation. For a doubly periodic flow in $L \times L$, the right-hand side is replaced by [52]

$$
\bar{w}_{j}=\frac{1}{2 i L} \sum_{k} \gamma_{k} \sum_{m^{\prime}=\infty}^{\infty} \cot \left(\frac{\pi\left(z_{j}-z_{k}\right)}{L}+i m \pi\right),
$$

where the inner sum includes $m=0$ except for $k=j$. In practice, this inner series can be truncated to $m \leqslant 2$. The system (A4) is integrated with a second-order Runge-Kutta method, which is formally symplectic for uniform time step $\Delta t$ [57]. The spatial resolution is determined by this time step. The critical case is when two corotating vortices get closer than a distance $\ell$, in which case they should rotate around each other with velocity $|w|=\gamma / 2 \pi \ell$. Accuracy requires that $|w| \Delta t \ll \ell$, or $\ell \gg(\gamma \Delta t / 2 \pi)^{1 / 2}$. The accuracy constraint is laxer for counterrotating pairs, which tend to translate in a straight line. For shorter distances, corotating and counterrotating pairs behave different for numerical reasons and there is an apparent correlation dip reminiscent of Fig. 4(c). This was tested by changing the time step by a factor of 4 , so that $\ell / \lambda_{P}=0.05-0.2$, and became the reason why T1024a was rerun at a half as long time step, without any effect.

\section{APPENDIX B: VORTEX OVERLAP}

Consider sets of $N$ circular vortices, of uniform diameter $R$, in a box of area $L^{2}$. Two vortices intersect if their centers are at a distance $h<2 R$ and the relevant parameter is $\sigma=h / 2 R \in(0,1)$. The area of their intersection is

$$
\frac{\Delta S}{2 R^{2}}=\arccos (\sigma)-\sigma\left(1-\sigma^{2}\right)^{1 / 2}
$$


The probability density of the center of a vortex being at a distance $h$ from another one is $2 \pi h / L^{2}$, so the average intersection area is

$$
\left\langle\Delta S_{1}\right\rangle=\int_{0}^{1} p(\sigma) \Delta S d \sigma=\frac{16 \pi R^{4}}{L^{2}} \int_{0}^{1} \sigma\left[\arccos (\sigma)-\sigma\left(1-\sigma^{2}\right)^{1 / 2}\right] d \sigma=\frac{\pi^{2} R^{4}}{L^{2}} .
$$

Since the total number of possible vortex pairs is $N(N-1) / 2$ and the total vortex area is $\pi N R^{2}$, the relative area fraction of the intersections is

$$
\frac{\left\langle\Delta S_{N}\right\rangle}{S_{N}}=\frac{(N-1) \pi R^{2}}{2 L^{2}} \approx \frac{0.5 S_{N}}{L^{2}} .
$$

The experimental factor is closer to 0.45 , but the relation holds very well, particularly for the circular Gaussian cores.

\section{APPENDIX C: VORTEX ENERGY}

Consider a system of $n_{v}$ compact vortices in a doubly periodic box of side $L$, assumed to be of radius $R \ll L$ and divided into equal numbers of positive and negative circulation $\pm \gamma$. A rough estimation of the rms velocity fluctuations that they generate follows from assuming their induced velocities to be independent variables whose individual variance is the average

$$
q_{1}^{\prime 2}=\frac{1}{L^{2}} \int_{L^{2}}\left(\frac{\gamma}{2 \pi|\boldsymbol{x}|}\right)^{2} d^{2} \boldsymbol{x} \approx \frac{1}{L^{2}} \int_{R}^{\ell_{0}}\left(\frac{\gamma}{2 \pi r}\right)^{2} 2 \pi r d r=\frac{\gamma^{2}}{2 \pi L^{2}} \log \left(\frac{\ell_{0}}{R}\right),
$$

where $\ell_{0}$ is an outer scale beyond which the $1 / r$ velocity law ceases to apply. The expected velocity variance, or kinetic energy, due to $n_{v}$ cores is of the order of

$$
q^{\prime 2}=q_{1}^{\prime 2} n_{v} \approx \frac{\gamma^{2} n_{v}}{2 \pi L^{2}} \log \left(\frac{\ell_{0}}{R}\right) .
$$

Note that the assumption in this equation, in the notation of the body of the paper, is that randomized vortices only have self-energy. If we similarly estimate the mean enstrophy as

$$
\omega^{\prime 2} \approx\left(\frac{\gamma}{R^{2}}\right)^{2} n_{v}\left(\frac{R}{L}\right)^{2}
$$

we obtain

$$
q^{\prime 2} /\left(\omega^{\prime 2} R^{2}\right) \sim \log \left(\frac{\ell_{0}}{R}\right)
$$

For regular lattices such as those in Sec. VI, $\ell_{0}=2\left(\Delta_{1} \Delta_{2}\right)^{1 / 2} / \pi$, where the $\Delta_{j}$ are the lengths of the two vectors that define the lattice cell [53]. For a random superposition of vortices in a periodic box, this becomes $\ell_{0}=2 L / \pi$ and the logarithmic correction in Fig. 4(b) applies. On the other hand, if the velocity is screened beyond distances proportional to $R$, the correction disappears.

[1] J. Jiménez, Machine-aided turbulence theory, J. Fluid Mech. 854, R1 (2018).

[2] J. Jiménez, Computers and turbulence, Eur. J. Mech. B 79, 1 (2020).

[3] J. Jiménez, Monte Carlo science, J. Turbul. 21, 544 (2020).

[4] J. Jiménez, Dipoles and streams in two-dimensional turbulence, J. Fluid Mech. 904, A39 (2020).

[5] G. K. Batchelor, Computation of the energy spectrum in homogeneous two dimensional turbulence, Phys. Fluids 12, II-233 (1969).

[6] G. Joyce and D. Montgomery, Negative temperature states for the two-dimensional guiding-centre plasma, J. Plasma Phys. 10, 107 (1973). 
[7] J. C. McWilliams, An application of equivalent modons to atmospheric blocking, Dyn. Atmos. Oceans 5, 43 (1980).

[8] R. Benzi, S. Patarnello, and P. Santangelo, On the statistical properties of decaying two-dimensional turbulence, Europhys. Lett. 3, 811 (1987).

[9] M. E. Maltrud and G. K. Vallis, Energy spectra and coherent structures in forced two-dimensional and beta-plane turbulence, J. Fluid Mech. 228, 321 (1991).

[10] L. Onsager, Statistical hydrodynamics, Nuovo Cimento Suppl. 6, 279 (1949).

[11] D. Montgomery and G. Joyce, Statistical mechanics of "negative temperature" states, Phys. Fluids 17, 1139 (1974).

[12] D. Montgomery, W. H. Matthaeus, W. T. Stribling, D. Martinez, and S. Oughton, Relaxation in two dimensions and the "sinh-Poisson" equation, Phys. Fluids A 4, 3 (1992).

[13] R. H. Kraichnan, Inertial ranges in two-dimensional turbulence, Phys. Fluids 10, 1417 (1967).

[14] R. H. Kraichnan, Inertial range transfer in two- and three-dimensional turbulence, J. Fluid Mech. 47, 525 (1971).

[15] J. C. McWilliams, A demonstration of the suppression of turbulent cascades by coherent vortices in twodimensional turbulence, Phys. Fluids A 2, 547 (1990).

[16] G. F. Carnevale, J. C. McWilliams, Y. Pomeau, J. B. Weiss, and W. R. Young, Evolution of Vortex Statistics in Two-Dimensional Turbulence, Phys. Rev. Lett. 66, 2735 (1991).

[17] R. Benzi, M. Colella, M. Briscolini, and P. Santangelo, A simple point vortex model for two-dimensional decaying turbulence, Phys. Fluids A 4, 1036 (1992).

[18] D. G. Dritschel, R. K. Scott, C. Macaskill, G. A. Gottwald, and C. V. Tran, Unifying Scaling Theory for Vortex Dynamics in Two-Dimensional Turbulence, Phys. Rev. Lett. 101, 094501 (2008).

[19] J. Paret and P. Tabeling, Intermittency in the two-dimensional inverse cascade of energy: Experimental observations, Phys. Fluids 10, 3126 (1998).

[20] G. Boffetta, A. Celani, and M. Vergassola, Inverse energy cascade in two-dimensional turbulence: Deviations from Gaussian behavior, Phys. Rev. E 61, R29 (2000).

[21] G. L. Eyink, Multiscale gradient expansion of the turbulent stress tensor, J. Fluid Mech. 549, 159 (2006).

[22] Z. Xiao, M. Wan, S. Chen, and G. L. Eyink, Physical mechanism of the inverse energy cascade of twodimensional turbulence: A numerical investigation, J. Fluid Mech. 619, 1 (2009).

[23] M. E. Maltrud and G. K. Vallis, Energy and enstrophy transfer in numerical simulations of twodimensional turbulence, Phys. Fluids A 5, 1760 (1993).

[24] V. Borue, Inverse Energy Cascade in Stationary Two-Dimensional Homogeneous Turbulence, Phys. Rev. Lett. 72, 1475 (1994).

[25] L. M. Smith and V. Yakhot, Bose Condensation and Small-Scale Structure Generation in a Random-Force Driven 2D Turbulence, Phys. Rev. Lett. 71, 352 (1993).

[26] L. M. Smith and V. Yakhot, Finite-size effects in forced two-dimensional turbulence, J. Fluid Mech. 274, 115 (1994).

[27] P. Tabeling, Two-dimensional turbulence: A physicist approach, Phys. Rep. 362, 1 (2002).

[28] G. Boffetta and R. E. Ecke, Two-dimensional turbulence, Annu. Rev. Fluid Mech. 44, 427 (2012).

[29] D. Ruelle, Is there screening in turbulence?, J. Stat. Phys. 61, 865 (1990).

[30] G. Kawahara, M. Uhlmann, and L. van Veen, The significance of simple invariant solutions in turbulent flows, Annu. Rev. Fluid Mech. 44, 203 (2012).

[31] J. Jiménez, Algebraic probability density functions in isotropic two-dimensional turbulence, J. Fluid Mech. 313, 223 (1996).

[32] G. K. Batchelor, An Introduction to Fluid Dynamics (Cambridge University Press, Cambridge, 1967), Sec. 7.3.

[33] K. A. O’Neil, On the Hamiltonian dynamics of vortex lattices, J. Math. Phys. 30, 1373 (1989).

[34] L. D. Landau and E. M. Lifshitz, Statistical Physics, 2nd ed. (Addison-Wesley, Reading, 1958).

[35] T. Ishida, P. A. Davidson, and Y. Kaneda, On the decay of isotropic turbulence, J. Fluid Mech. 564, 455 (2006).

[36] P. A. Davidson, Long-range interactions in turbulence and the energy decay problem, Philos. Trans. R. Soc. A 369, 796 (2011). 
[37] H. Aref, P. K. Newton, M. A. Stremler, T. Tokieda, and D. L. Vainchtein, Vortex crystals, Adv. Appl. Mech. 39, 1 (2003).

[38] J. C. R. Hunt and D. J. Carruthers, Rapid distortion theory and the 'problems' of turbulence, J. Fluid Mech. 212, 497 (1990).

[39] P. W. Terry, Suppression of turbulence and transport by shear flow, Rev. Mod. Phys. 72, 109 (2000).

[40] J. C. R. Hunt, I. Eames, and J. Westerweel, Mechanics of inhomogeneous turbulence and interfacial layers, J. Fluid Mech. 554, 499 (2006).

[41] P. Meunier, S. Le Dizès, and T. Leweke, Physics of vortex merging, C. R. Phys. 6, 431 (2005).

[42] G. R. Flierl, V. D. Larichev, J. C. McWilliams, and G. M. Reznik, The dynamics of baroclinic and barotropic solitary eddies, Dyn. Atmos. Oceans 5, 1 (1980).

[43] D. W. Moore and P. G. Saffman, Structure of a line vortex in an imposed strain, in Aircraft Wake Turbulence and its Detection, edited by J. H. Olsen, A. Goldburg, and M. Rogers (Springer, Berlin, 1971), pp. 339-354.

[44] D. W. Moore and P. G. Saffman, The density of organized vortices in a turbulent mixing layer, J. Fluid Mech. 69, 465 (1975).

[45] S. Chandrasekhar, The equilibrium and stability of the Roche ellipsoids, Astrophys. J. 138, 1182 (1963).

[46] M. S. Nikulin, Hellinger distance, Encyclopedia of Mathematics (EMS, Berlin, 2001).

[47] D. Stoyan, W. S. Kendall, and J. Mecke, Stochastic Geometry and its Applications (Wiley, New York, 1987).

[48] K. S. Fine, A. C. Cass, W. G. Flynn, and C. F. Driscoll, Relaxation of 2D Turbulence to Vortex Crystals, Phys. Rev. Lett. 75, 3277 (1995).

[49] D. Z. Jin and D. H. E. Dubin, Characteristics of Two-Dimensional Turbulence that Self-Organizes into Vortex Crystals, Phys. Rev. Lett. 84, 1443 (2000).

[50] J. Jiménez and A. Guegan, Spontaneous generation of vortex crystals from forced two-dimensional homogeneous turbulence, Phys. Fluids 19, 085103 (2007).

[51] F. Tabataba-Vakilia, J. H. Rogers, G. Eichstädt, G. S. Orton, C. J. Hansen, T. W. Momary, J. A. Sinclair, R. S. Giles, M. A. Caplinger, M. A. Ravine, and S. J. Bolton, Long-term tracking of circumpolar cyclones on Jupiter from polar observations with JunoCam, Icarus 335, 113405 (2020).

[52] H. Lamb, Hydrodynamics, 6th ed. (Cambridge University Press, Cambridge, 1932).

[53] V. K. Tkachenko, On vortex lattices, Zh. Éksp. Teor. Fiz. 49, 1875 (1965) [Sov. Phys. JETP 22, 1282 (1966)].

[54] J. Jiménez, On the linear stability of the inviscid Kármán vortex street, J. Fluid Mech. 178, 177 (1987).

[55] V. K. Tkachenko, Stability of vortex lattices, Zh. Éksp. Teor. Fiz. 50, 1573 (1966) [Sov. Phys. JETP 23, 1049 (1966)].

[56] D. E. Sanders, Introduction to Crystallography (Dover, New York, 1993).

[57] J. M. Sanz-Serna and M. P. Calvo, Numerical Hamiltonian Problems (Chapman Hall, London, 1994). 